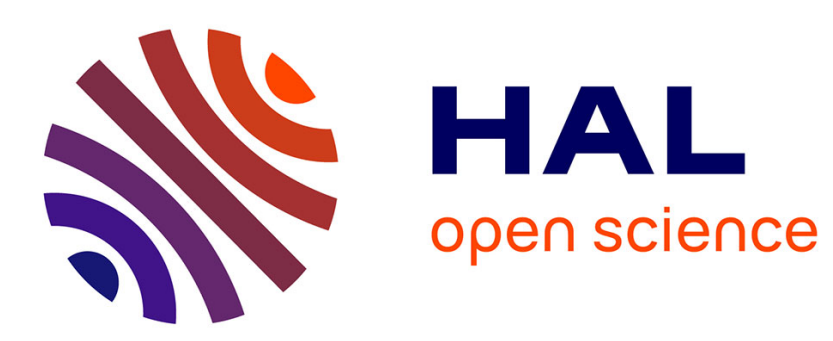

\title{
Cartan's moving frame method and its application to the geometry and evolution of curves in the Euclidean, affine and projective planes
}

Olivier Faugeras

\section{- To cite this version:}

Olivier Faugeras. Cartan's moving frame method and its application to the geometry and evolution of curves in the Euclidean, affine and projective planes. [Research Report] RR-2053, INRIA. 1993. inria-00074619

\section{HAL Id: inria-00074619 \\ https://hal.inria.fr/inria-00074619}

Submitted on 24 May 2006

HAL is a multi-disciplinary open access archive for the deposit and dissemination of scientific research documents, whether they are published or not. The documents may come from teaching and research institutions in France or abroad, or from public or private research centers.
L'archive ouverte pluridisciplinaire HAL, est destinée au dépôt et à la diffusion de documents scientifiques de niveau recherche, publiés ou non, émanant des établissements d'enseignement et de recherche français ou étrangers, des laboratoires publics ou privés. 
Cartan's Moving Frame Method and its Application to the Geometry and Evolution of Curves in the Euclidean, Affine and Projective Planes

Olivier FAUGERAS

$N^{\circ} 2053$

Octobre 1993

PROGRAMME 4

Robotique, image

et

vision

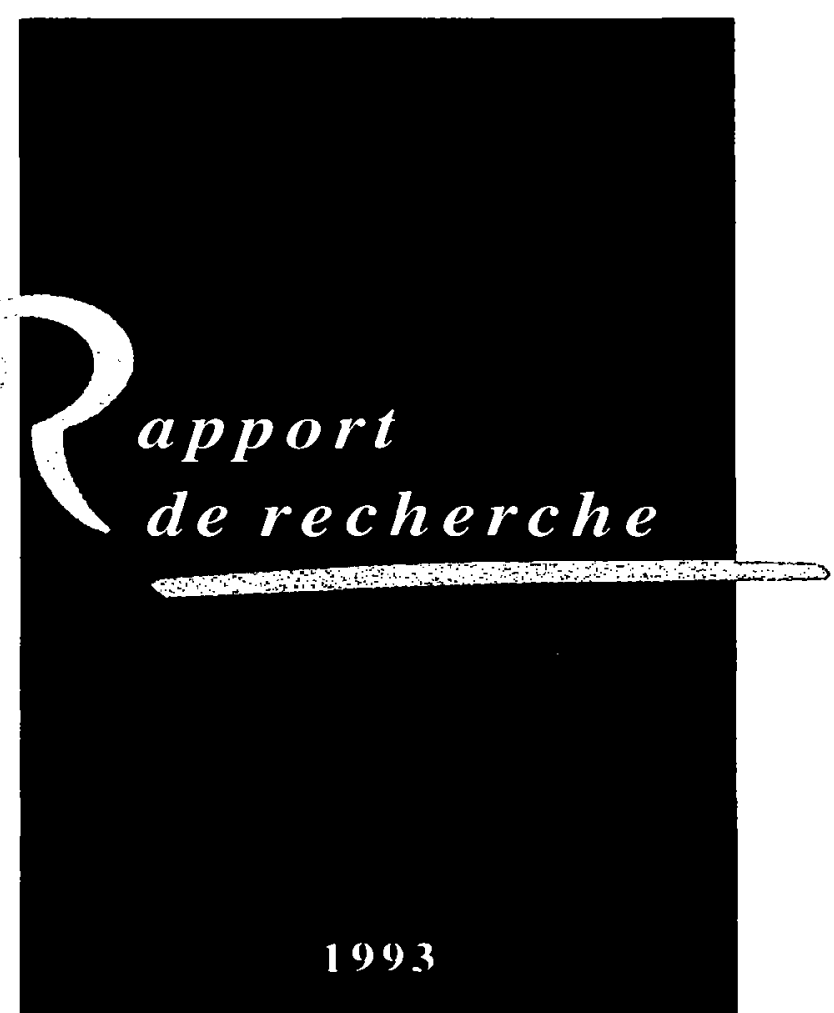




\title{
La méthode du repère mobile de Cartan et son application à l'étude de la géométrie et de l'évolution des courbes planes euclidiennes, affines et projectives
}

\author{
Olivier Faugeras
}

\begin{abstract}
Cet article est une introduction générale à la méthode du repère mobile de Cartan, méthode qui est élégante, simple et de nature algorithmique. Nous montrons comment l'utiliser de manière systématique sur trois examples qui sont importants pour la vision par ordinateur, à savoir les courbes planes des plans euclidien, affine et projectif pour calculer les équations de Frenet correspondantes. Nous utilisons ensuite ces équations pour montrer comment l'analyse de la déformation d'une courbe plane selon une équation de la chaleur intrinsèque peut se faire dans un cadre uniforme, et nous obtenons des expressions très voisines décrivant l'évolution des trois invariants de courbure.
\end{abstract}

\section{Cartan's moving frame method and its application to the geometry and evolution of curves in the euclidean, affine and projective planes}

\begin{abstract}
This article is a general introduction to Cartan's moving frame method which is elegant, simple, and of an algorithmic nature. We have demonstrated how to use it systematically on three examples relevant to computer vision, curves in the euclidean, affine and projective planes, and derived the corresponding Frenet equations. We have then used these equations to show that the analysis of the deformation of plane curves according to an intrinsic heat equation could be done in a common framework, yielding very similar expressions for the evolution of the three curvature invariants.
\end{abstract}




\section{Introduction and motivation}

A renewed interest in the theory of invariants has developed in the vision and robotics communities speared by the need to increase the capabilities of artificial systems to represent objects shapes and their robustness in performing recognition tasks. This has produced a large number of publications and a book [16]. It is not too surprising that the question of invariance is also at the heart of another problem central to computer vision, namely the problem of scale-space analysis. These two questions, namely invariant shape representation and scale-space are at he heart of the core of Koenderink and his coworkers $[14,13,7]$.

This article addresses the question of describing the differential properties of shapes which are invariant to the action of a group. The shapes of interest are differentiable manifolds such as curves and surfaces but can also be differentiable sets of lines such as complexes or congruences. The groups of interest in computer vision are the euclidean, affine (or unimodular affine) and projective groups.

Among the methods that can be used to obtain such descriptions there is one that clearly emerges because of its simplicity, elegance, generality, and because it is quite amenable to computer implementation. This method is known as the Cartan's moving frame method and has been developed in the first decades of this century by Elie Cartan and his students [2,3]. The method is widely used in mathematics and physics but has not yet attracted many researchers in computer vision with the notable exception of ter Haar Romeny and his coworkers [17].

In section 2 of this article we give a detailed description of the moving frame method which is completely general and can be used (and automated) in all practical cases. This description uses the tools of the modern exterior differential calculus which were being invented at the time Cartan was developing his moving frame method and is an extended version of what can be found in [2].

We then attempt to help the reader develop some intuition about how the method actually works by using it on three simple and useful examples: plane curves subject to the action of the euclidean, affine, and projective groups. To help even further the intuition we present geometric interpretations of the affine and projective arc lengths. We also relate projective and affine invariants to the more familiar euclidean ones. We found these relations quite useful in applications.

The question of scale-space is discussed in the context of curve evolution. This approach is closely related to the scale-space defined for grey-scale images [23] through for example the evolution of isophotes. But it is also closely related to the question of shape description as shown for example in the work of Kimia Tannenbaum and Zucker [12]. For the three groups of interest we show that the Cartan's method provides a natural framework in which to think about curve evolution as predicted by an intrinsic heat equation. One important idea which follows from the study of differential invariants is that the evolution of the curve is defined, up to a transformation of the group of interest, by the evolution of its arc length and of its curvature. The euclidean case had been covered by Gage and Hamilton $[8,9,10]$ from the viewpoint of mathematicians and by Mackworth and Mokhtarian in computer vision [15]. The affine case has been worked out by Sapiro and Tannenbaum [19, 18, 21, 20] and by Alvarez, Guichard, Lions and Morel [1]. The projective case is new and we shed some light on it in this article.

One interesting fact is that the three scale-spaces are intimately connected in the sense that they can be thought of as forming a hierarchy. It is likely that this hierarchy can be used in several ways. In this article we develop only the application that consists in reducing the order of derivation that is required to compute the invariants. For example, we show that the affine scale-space reduces the 
order of derivation necessary to compute the affine curvature from four to three by trading space for scale. A similar property holds in the projective case.

\section{Method of moving frames}

The method of moving frames, due to Elie Cartan [2,3], deals in a very simple way with problems of differential calculus on curves and surfaces in a context which is not necessarily the usual Euclidean one. In our opinion, the efficiency of the method lies in its potential for being automated, i.e., we can easily write it in an algorithmic form.

We will consider essentially projective, affine and Euclidean spaces which can be regarded as specialisations of each other. Groups of the same name are fundamental for understanding these spaces.

\subsection{Background on projective frames}

A projective frame in $\mathcal{P}^{n}$ is a set of $n+2$ projective points such that no subset of $n+1$ of these points belongs to the same hyperplane. We will designate them by $A_{0}, A_{1}, \cdots, A_{n+1}$. If we choose $A_{n+1}$ such that

$$
\mathbf{A}_{n+1}=\sum_{i=0}^{n} \mathbf{A}_{i}
$$

then the representatives of points $A_{i}, i=0, \cdots, n$ are defined up to global scale, i.e., if $\mathbf{A}_{i}$ is a representative of point $A_{i}$, then so is $\lambda \mathbf{A}_{i}$, for any $i=0, \cdots, n$.

With this choice, the fact that no subset of $n+1$ of these points belongs to the same hyperplane is equivalent to saying that the determinant of size $(n+1) \times(n+1)$ of representatives of the first $n+1$ is different from 0

$$
\operatorname{det}\left(\mathbf{A}_{0}, \cdots, \mathbf{A}_{n}\right) \neq 0
$$

The set of frames of the vector space $R^{n+1}$ satisfying the previous condition is an open set $U$ of $\left(R^{n+1}\right)^{n+1}$, the complement of the closed set defined by

$$
\operatorname{det}\left(\mathbf{A}_{0}, \cdots, \mathbf{A}_{n}\right)=0
$$

We can normalize things in such a way that we have the important condition

$$
\operatorname{det}\left(\mathbf{A}_{0}, \cdots, \mathbf{A}_{n}\right)=1
$$

The mappings $A_{i}: U \rightarrow R^{n+1}$ will be considered as the differentiable mappings of the open set of $\left(R^{n+1}\right)^{n+1}$ into $R^{n+1}$. For a given frame $r$, we note $\mathbf{A}_{i}(r)$ the value of that function at the point $r$ of $U$, i.e. the representative of the $i+$ first point of the frame $r$.

\subsection{Cartan equations: projective case}

We will consider a frame $r \in U$ depending on one or more parameters. Such a frame is called a moving frame.

The $n+1$ functions $A_{0}, A_{1}, \cdots, A_{n}$ defined on the open set $U$ naturally give us $n$ differential forms of degree one. Therefore $d \mathbf{A}_{i}(r) \in \mathcal{L}\left(\left(R^{n+1}\right)^{n+1} ; R^{n+1}\right)$, the set of linear mappings from $\left(R^{n+1}\right)^{n+1}$ 
into $R^{n+1}$. One of the key points of the method of moving frames is to write the value of this linear mapping in the projective frame $r$

$$
d \mathbf{A}_{i}(r)=\sum_{j=0}^{n} \omega_{i j}(r) \mathbf{A}_{j}(r)
$$

Therefore the functions $\omega_{i j}$ are differential forms of degree one with values in $R$.. This relation is true for $i=0, \cdots, n+1$.

The differential forms $\omega_{i j}$ are not independent, they satisfy a number of equations, called the Cartan or Lie equations which we now derive.

By differentiating the condition (1) on the determinant of points $A_{i}$, we immediately obtain the following relation satisfied by the forms $\omega_{i j}$

$$
\sum_{i=0}^{n} \omega_{i i}=0
$$

This means that there are only $(n+1)^{2}-1$ independent differential forms. This is precisely the dimension of the projective group of $\mathcal{P}^{n}$. By writing that the second differentials of forms $d \mathbf{A}_{i}$ are zero we obtain $n+1$ additional relations which must be satisfied by the forms $\omega_{i j}$. In effect, from well-known properties of exterior differentiation, these relations can be written

$$
d\left(d \mathbf{A}_{i}\right)=\sum_{j=0}^{n}\left(d \mathbf{A}_{j} \wedge \omega_{i j}+d \omega_{i j} \mathbf{A}_{j}\right)=0
$$

If we replace $d \mathbf{A}_{j}$ in this equation by that given in (2), we obtain the following relations

$$
\mathbf{0}=\sum_{j=0}^{n}\left(d \omega_{i j}-\sum_{i=0}^{n} \omega_{i k} \wedge \omega_{k j}\right) \mathbf{A}_{j} \quad i=0, \cdots, n
$$

and since the points $A_{j}$ form a projective frame, we deduce

$$
d \omega_{i j}=\sum_{k=0}^{n} \omega_{i k} \wedge \omega_{k j}
$$

These equations, called the structure or Cartan equations, are fundamental for the study of projective curves and surfaces.

\subsection{Cartan equations: Affine and Euclidean cases}

This is a specialization of the previous ideas to the affine and Euclidean cases. We will need coordinates and we denote by $x_{0}, \cdots, x_{n}$ the coordiantes of a point of $\mathcal{P}^{n}$. In the first case, we choose a particular hyperplane $H$ of $\mathcal{P}^{n}$ to which we can give an affine space structure as the hyperplane at infinity of space $\mathcal{P}^{n}$. The affine transformations constitute the subgroup of the projective group that leaves $H$ invariant. The points of $H$ represent the directions of the affine space $\mathcal{A}^{n}$ thus constructed. If we choose for $H$ the hyperplane of equation $x_{n}=0$, then a point of $\mathcal{A}^{n}$ with coordinates $x_{0}, \cdots, x_{n-1}$ corresponds to the point of $\mathcal{P}^{n}$ with coordinates $x_{0}, \cdots, x_{n-1}, 1$ and a vector $\mathrm{v}$ of $R^{n}$ corresponds to the point of $H$ with coordinates $\left[\mathbf{v}^{T}, 0\right]^{T}$, to within a nonzero scalar. 
An affine frame consists of a point $A$, the origin of the frame, and $n$ vectors of $R^{n}$ written $\mathbf{e}_{i}, i=1, \cdots, n$. We suppose that these vectors are linearly independent, i.e.,

$$
\operatorname{det}\left(\mathbf{e}_{\mathbf{i}}\right) \neq 0
$$

An affine frame can thus be considered as a particular projective frame for which $A_{0}$ is in $\mathcal{P}^{n} \backslash H$ and the $A_{i}, i=1, \cdots, n$ are in $H$. Condition (6) implies that the set of affine frames is an open set $U$ of $R^{n(n+1)}$. $A$ and $\mathbf{e}_{i}$ are thus differentiable functions $U \rightarrow R^{n}$. As in the projective case, we can consider an affine frame $r$ which depends on one or more parameters. This frame is called moving frame. Using reasoning similar to the projective case allows us to write the differential forms $d A, d e_{1}, \cdots, d e_{n}$ as linear combinations of vectors $e_{i}$

$$
\begin{aligned}
d \mathbf{A} & =\sum_{i=1}^{n} \omega_{i} \mathbf{e}_{i} \\
d \mathbf{e}_{i} & =\sum_{i=1}^{n} \omega_{i j} \mathbf{e}_{j}
\end{aligned}
$$

In effect, we have the particular case $\mathbf{A}_{0}=\left[\mathbf{A}^{T}, 1\right]^{T}, \mathbf{A}_{i}=\left[\mathbf{e}_{i}^{T}, 0\right]^{T}, i=1, \cdots, n$, thus $d \mathbf{A}$ and $d \mathbf{e}_{i}$ only depend on $\mathbf{e}_{i}, i=1, \cdots, n$.

Equations (5) are still valid

$$
\begin{aligned}
d \omega_{i} & =\sum_{k=1}^{n} \omega_{k} \wedge \omega_{k i} \\
d \omega_{i j} & =\sum_{k=1}^{n} \omega_{i k} \wedge \omega_{k j} \quad i, j=1, \cdots, n
\end{aligned}
$$

If we consider the subset of affine frames such that $\operatorname{det}\left(\mathbf{e}_{i}\right)=\operatorname{det}\left(\mathbf{A}_{0}, \cdots, \mathbf{A}_{n}\right)=1$, we must add an equation similar to equation (3)

$$
\sum_{i=1}^{n} \omega_{i i}=0
$$

Corresponding to this restriction on frames is a restriction on the group of transformations of the space in which we are interested. Here we consider the unimodal subgroup of the affine group. This subgroup is defined as follows. The transformation $M^{\prime}$ of a point $M$ of $\mathcal{A}^{n}$ by an affine tranformation is given by the relation

$$
\mathbf{M}^{\prime}=\mathbf{H M}+\mathbf{b}
$$

where $\mathbf{H}$ is a matrix of size $n \times n$ such that $\operatorname{det}(\mathbf{H}) \neq 0$, and $\mathbf{b}$ is a vector of size $n$. The unimodal subgroup is the one for which we have $\operatorname{det}(\mathbf{H})=1$. The unimodular affine group depends upon $n^{2}-1+1$ parameters which, according to ( 9 is precisely the number of independent differential forms $\omega_{i}$ and $\omega_{i j}$.

We consider the Euclidean case similarly by introducing Euclidean frames which are affine frames such that the matrix of the vectors $e_{i}$ is orthogonal. We express it in the form

$$
\mathbf{e}_{i} \cdot \mathbf{e}_{j}=\delta_{i j}
$$


and by differentiating these relations, we obtain

$$
\mathbf{e}_{i} \cdot d \mathbf{e}_{j}+d \mathbf{e}_{i} \cdot \mathbf{e}_{j}=0
$$

We deduce by replacing the differentials with respect to $\omega_{i j}$

$$
\omega_{i j}+\omega_{j i}=0 i, j=1, \cdots, n
$$

and thus we have in particular

$$
\omega_{i i}=0 \quad \forall i
$$

The euclidean group depends upon $q=\frac{n^{2}+n}{2}$ parameters which, according to our analysis, is precisely the number of independent differential forms $\omega_{i}$ and $\omega_{i j}$.

\subsection{Introduction to the method of moving frames}

We will consider a set $D$ of points operated on by a finite and continuous group $G$ with $q$ parameters. In practice, $D$ will be a Euclidean, affine, or projective space and $G$ will be one of the groups previously studied. In this section, we note $\omega_{1}, \cdots, \omega_{q}$ the differential forms attached to a general frame.

We will consider manifolds of dimension $\lambda$ in $D$. In practice, we have $\lambda=1$ or 2 , i.e., we will study curves or surfaces. To a point $A$ of manifold $V_{\lambda}$ we attach two types of elements

Frames : an infinity of inclusive families of frames: frames of order $0, \cdots$, frames of order $P$.

Integers : $\mu_{0}$, the number of invariants of order $0, \cdots, \mu_{P}-\mu_{P-1}$, the number of invariants of order $P$.

We then define the contact element of order $P$ of the point $A$ of $V_{\lambda}$ as the family of frames of order $P$ of this point and by the set of invariants of order $\leq P$ of this point. Note that the transformations of these frames into each other constitute a subgroup $\mathcal{G}_{P}$ of $G$, called the subgroup of order $P$ of the contact element considered.

In other words, each contact element is characterized by a family of frames relative to which it occupies the same position, and by a system of numbers concerning its "form", the invariants. This notion of families of included frames is interesting for many reasons. One of them is that it will allow us to compare the Frenet frames obtained for groups of included transformations: the Frenet frame for the Euclidean group will be a particular frame of order three for the affine group. Similarly, the affine Frenet frame is a particular frame of order 3 for the projective group.

Let us take a contact element of order $P$; its most general frame of order $P$ depends on $\nu_{P}$ parameters, which we call secondary parameters of order $P$ and write $x_{1}, \cdots, x_{\nu_{P}}$. These parameters are different from the $\lambda$ parameters, called principal parameters, on which depend the points of the manifold $V_{\lambda}$ and which are written $t_{1}, \cdots, t_{\lambda}$. Note that the frames of a contact element correspond to constant values of principal parameters since the origin $A$ of the frame is fixed. We can show that infinitesimal translations of this frame are characterized by the property that $q-\nu_{P}$ linear combinations of the $\omega_{i}$ components disappear. More precisely, infinitesimal translations of the most general frame of order $P-1$, attached to the contact element being considered, eliminate $q-\nu_{P-1}$ 
independent linear combinations of $\omega_{1}, \cdots, \omega_{q}$. In effect, for a frame of order $P-1$, the forms $\omega_{1}, \cdots, \omega_{q}$ depend on $\lambda+\nu_{P-1}$ parameters and we can write

$$
\omega_{i}=\sum_{j=1}^{\lambda} a_{i j} d t_{j}+\sum_{k=1}^{\nu_{P-1}} b_{i k} d x_{k}
$$

where $a_{i j}$ and $b_{i k}$ are functions of $t_{1}, \cdots, t_{\lambda}, x_{1}, \cdots, x_{\nu_{P-1}}$. By eliminating the $\nu_{P-1}$ forms $d x_{k}$ from among the $q$ forms $\omega_{i}$, we obtain $q-\nu_{P_{-1}}$ linear combinations of the $q$ forms $\omega_{i}$ which are expressed as linear combinations of the $\lambda$ forms $d t_{j}$ and which disappear when the principal parameters are constant, that is for a frame belonging to a contact element of order $P-1$, or in fact, to a contact element of any order higher than $P-1$.

The infinitesimal translation of a frame of order $P$ eliminates these $q-\nu_{P-1}$ linear combinations and $\nu_{P-1}-\nu_{P}$ others that we will call principal components of order $P$. We note them $\pi_{\alpha}, \alpha=$ $q-\nu_{P-1}+1, q-\nu_{P}$.

Let us now consider the most general contact element of order 0 , i.e., any point $A$ of $D$ and vertex frames of this point. Reasoning similar to the above shows that the principal parameter differentials are linear combinations of $\lambda$ forms suitably chosen among the principal components of order 0 . We assume in the following, to simplify without loss of generality, that these $\lambda$ forms are the first $\lambda$ principal components of order 0 , of which there are $q-\nu_{0}$, i.e., $\pi_{1}, \cdots, \pi_{\lambda}$.

A frame which varies while always remaining of order $P$ depends on the $\lambda$ principal parameters of $V_{\lambda}$ and on $\nu_{P}$ secondary parameters of order $P$. By definition, its principal components of orders $\leq P$ are independent of the differentials of the secondary parameters; these are thus linear combinations of $\pi_{1}, \cdots, \pi_{\lambda}$. Therefore, we have the following proposition

Proposition 2.1 The principal components of orders $<P$ of the frames of order $P$ of $V_{\lambda}$ are liricar combinations of $\pi_{1}, \cdots, \pi_{\lambda}$ with coefficients in terms of invariants of order $\leq P$.

Since the invariants are functions of the principal parameters only, we can also state the following proposition

Proposition 2.2 The differentials of invariants of orders $<P$ are linear combinations of $\pi_{1}, \cdots, \pi_{\lambda}$ with coefficients which are functions of the invariants of orders $\leq P$. The differentials of the invariants of order $P$ and the principal components of order $P$ of the frames of order $P$ are thus linear combinations of $\pi_{1}, \cdots, \pi_{\lambda}$ with coefficients in terms of the invariants of order $\leq P$

$$
\begin{array}{r}
d k_{\alpha}=\sum_{i=1}^{\lambda} b_{\alpha i} \pi_{i} \mu_{P-1}<\alpha \leq \mu_{P} \\
\pi_{\alpha}=\sum_{i=1}^{\lambda} b_{\alpha i}^{\prime} \pi_{i} q-\nu_{P-1}<\alpha \leq q-\nu_{P}
\end{array}
$$

The coefficients $b_{\alpha i}$ and $b_{\alpha i}^{\prime}$ are functions of the invariants of order $\leq P$ and of the secondary parameters of order $P$. They are called coefficients of order $P$. The fundamental idea of the method of moving frames is to study how the group $\mathcal{G}_{P}$ affects coefficients of order $P$. 
Let $\pi_{i}, i=1, \cdots, q-\nu_{P}$ be the $q-\nu_{P}$ principal components of order $P$ which are linear combinations of $q$ forms $\omega_{i}$

$$
\pi_{i}=\sum_{j=1}^{q} a_{i j} \omega_{j} \quad i=1, \cdots, q-\nu_{P}
$$

The coefficients $a_{i j}$ are either constants or functions of invariants or order $\leq P$. Let us compute the exterior differentials of the $\pi_{i}$

$$
d \pi_{i}=\sum_{j=1}^{q} d a_{i j} \wedge \omega_{j}+\sum_{j=1}^{q} a_{i j} d \omega_{j}
$$

Differentials of $a_{i j}$ can be written in the form

$$
d a_{i j}=\sum_{m=1}^{\mu_{P}} D_{i j m} d k_{m}
$$

and we know the structure equations of group $G$

$$
d \omega_{p}=\sum_{s, t} c_{p o t} \omega_{s} \wedge \omega_{t}
$$

To simplify the developement we then suppose that the rank of the first square submatrix of size $q-\nu_{P}$ of the matrix $\left[a_{i j}\right]$ equals $q-\nu_{P}$ which allows us to compute $\omega_{i}, i=1, \cdots, q-\nu_{P}$ with respect to $\pi_{i}$

$$
\omega_{i}=\sum_{j=1}^{q-\nu_{P}} a_{i j}^{\prime} \pi_{j}+\sum_{n=1}^{\nu_{P}} d_{i n} \omega_{q-\nu_{P}+n} \quad i=1, \cdots, q-\nu_{P}
$$

If we now consider the differential system

$$
\pi_{1}=0 \cdots \pi_{q-\nu_{P}}=0
$$

this system is completely integrable when the principal parameters are constant. Therefore, according to Frobenius's theorem, we have the following relations

$$
d \pi_{i} \wedge \pi_{1} \wedge \cdots \wedge \pi_{q-\nu_{P}}=0 \quad i=1, \cdots, q-\nu_{P}
$$

By replacing $d \pi_{i}$ by its value (13) in this expression we obtain the relations

$$
\begin{gathered}
\sum_{j=1}^{q} d a_{i j} \wedge \omega_{j} \wedge \pi_{1} \wedge \cdots \wedge \pi_{q-\nu_{P}}+\sum_{s, t}\left(\sum_{j=1}^{q} a_{i j} c_{j s t}\right) \omega_{s} \wedge \omega_{t} \wedge \pi_{1} \wedge \cdots \wedge \pi_{q-\nu_{P}}=0 \\
i=1, \cdots, q-\nu_{P}
\end{gathered}
$$

The first part of this relation including the terms in $d a_{i j}$ identically equals zero, by (14) and (10). The other part will contribute to eliminating all terms containing factors $\omega_{s} \wedge \omega_{t}$ with $s$ and $t>q-\nu_{p}$. We can thus write

$$
\begin{gathered}
d \pi_{i}=\sum_{\alpha, \beta=1}^{q-\nu_{P}} C_{i \alpha \beta} \pi_{\alpha} \wedge \pi_{\beta}+\sum_{\alpha=1}^{q-\nu_{P}} \sum_{n=1}^{\nu_{P}} A_{i n \alpha} \pi_{\alpha} \wedge \omega_{q-\nu_{P}+n}+\sum_{m=1}^{\mu_{P}} \sum_{\alpha=1}^{q-\nu_{P}} D_{i m \alpha} d k_{m} \wedge \pi_{\alpha} \\
+\sum_{m=1}^{\mu_{P}} \sum_{n=1}^{\nu_{P}} B_{i m n} d k_{m} \wedge \omega_{q-\nu_{P}+n} i=1, \cdots, q-\nu_{P}
\end{gathered}
$$


We now reach the climax of the plot. Let us take the exterior differentials of equations (10) and (11)

$$
\begin{array}{r}
0=\sum_{i=1}^{\lambda} d b_{\alpha i} \wedge \pi_{i}+\sum_{i=1}^{\lambda} b_{\alpha i} \wedge d \pi_{i} \quad \mu_{P-1}<\alpha \leq \mu_{P} \\
d \pi_{\alpha}=\sum_{i=1}^{\lambda} d b_{\alpha i}^{\prime} \wedge \pi_{i}+\sum_{i=1}^{\lambda} b_{\alpha i}^{\prime} \wedge d \pi_{i} \quad q-\nu_{P-1}<\alpha \leq q-\nu_{P}
\end{array}
$$

and make two substitutions.

The first substitution consists of replacing the $\nu_{P}-\nu_{P-1}$ principal components $\pi_{\alpha}$ of order $P$ in the equations (16) by their values given by (11), thus depending on coefficients of order $P$ which we represent as the vector $\mathbf{b}^{\prime}$ of size $\lambda\left(\nu_{P}-\nu_{P-1}\right)$, the $q-\nu_{P-1}$ principal components of order $<P$ by their values with respect to $\pi_{1}, \cdots, \pi_{\lambda}$ and coefficients of order $<P$. Similarly, let us replace in these equations the differentials of the $\mu_{P}-\mu_{P-1}$ invariants of order $P$ by their values taken from (10), that is with respect to coefficients of order $P$ which we represent as the vector $b$ of size $\lambda\left(\mu_{P}-\mu_{P-1}\right)$, and the $\mu_{P-1}$ differentials of invariants of order $<P$ by their expressions with respect to $\pi_{1}, \cdots, \pi_{\lambda}$ and coefficients of order $<P$. We thus obtain

$$
\begin{gathered}
d \pi_{i}=\sum_{\alpha, \beta=1}^{\lambda} E_{i \alpha \beta}^{\prime}\left(\mathbf{b}, \mathbf{b}^{\prime}\right) \pi_{\alpha} \wedge \pi_{\beta}+\sum_{\alpha=1}^{\lambda} \sum_{n=1}^{\nu_{P}} A_{i \alpha n}^{\prime}\left(\mathbf{b}^{\prime}\right) \pi_{\alpha} \wedge \omega_{q-\nu_{P}+n}+ \\
\sum_{\alpha=1}^{\lambda} \sum_{n=1}^{\nu_{P}} B_{i \alpha n}^{\prime}(\mathbf{b}) \pi_{\alpha} \wedge \omega_{q-\nu_{P}+n}
\end{gathered}
$$

or

$$
d \pi_{i}=\sum_{\alpha, \beta=1}^{\lambda} E_{i \alpha \beta}^{\prime}\left(\mathbf{b}, \mathbf{b}^{\prime}\right) \pi_{\alpha} \wedge \pi_{\beta}+\sum_{\alpha=1}^{\lambda} \sum_{n=1}^{\nu_{P}} F_{i \alpha n}^{\prime}\left(\mathbf{b}, \mathbf{b}^{\prime}\right) \pi_{\alpha} \wedge \omega_{q-\nu_{P}+n} i=1, \cdots, q-\nu_{P}
$$

The second substitution consists of replacing the $d \pi_{i}$ by their values in equations (17) and (18). We then obtain

$$
\begin{aligned}
& \sum_{j=1}^{\lambda}\left(d b_{\alpha j}-\sum_{n=1}^{\nu_{P}} f_{\alpha j n}\left(\mathbf{b}, \mathbf{b}^{\prime}\right) \omega_{q-\nu_{P}+n}+\sum_{h=1}^{\lambda} r_{\alpha h}\left(\mathbf{b}, \mathbf{b}^{\prime}\right) \pi_{h}\right) \wedge \pi_{j}=0 \\
& \sum_{j=1}^{\lambda}\left(d b_{\alpha j}^{\prime}-\sum_{n=1}^{\nu_{P}} g_{\alpha j n}\left(\mathbf{b}, \mathbf{b}^{\prime}\right) \omega_{q-\nu_{P}+n}+\sum_{h=1}^{\lambda} r_{\alpha h}^{\prime}\left(\mathbf{b}, \mathbf{b}^{\prime}\right) \pi_{h}\right) \wedge \pi_{j}=0
\end{aligned}
$$

From these relations we deduce that

$$
d b_{\alpha j}-\sum_{n=1}^{\nu_{P}} f_{\alpha j n}\left(\mathbf{b}, \mathbf{b}^{\prime}\right) \omega_{q-\nu_{P}+n}+\sum_{h=1}^{\lambda} r_{\alpha h}\left(\mathbf{b}, \mathbf{b}^{\prime}\right) \pi_{h}
$$

and

$$
d b_{\alpha j}^{\prime}-\sum_{n=1}^{\nu_{P}} g_{\alpha j n}\left(\mathbf{b}, \mathbf{b}^{\prime}\right) \omega_{q-\nu_{P}+n}+\sum_{h=1}^{\lambda} r_{\alpha h}^{\prime}\left(\mathbf{b}, \mathbf{b}^{\prime}\right) \pi_{h}
$$

are linear combinations of $\lambda$ differential forms $\pi_{i}$. If we denote by $e_{q-\nu_{P}+n}$ the restriction of the differential form $\omega_{q-\nu_{P}+n}$ when the principal parameters are constant and the principal forms $\pi_{1}, \cdots, \pi_{\lambda}$ 
of order 0 disappear, we finally obtain differential equations yielding the action of the group $\mathcal{G}_{P}$ on coefficients of order $P$

$$
\begin{aligned}
& d b_{\alpha j}=\sum_{n=1}^{\nu_{P}} f_{\alpha j n}\left(\mathbf{b}, \mathbf{b}^{\prime}\right) e_{q-\nu_{P}+n} \\
& d b_{\alpha j}^{\prime}=\sum_{n=1}^{\nu_{P}} g_{\alpha j n}\left(\mathbf{b}, \mathbf{b}^{\prime}\right) e_{q-\nu_{P}+n}
\end{aligned}
$$

Let us examine in more detail how this leads to an algorithmic method for computing the different classes of frames. At the beginning of step $P+1$, we assume known

1. The number of invariants of order $1,2, \cdots, \mathrm{P}$;

2. The definition of frames of order $1,2, \cdots, P$;

3. The definition of principal components of orders $<P$ of frames of order $P$, their expressions in terms of $\pi_{1}, \cdots, \pi_{\lambda}$, and invariants of orders $\leq P$;

4. The expressions of differentials of invariants of orders $<P$ with respect to $\pi_{1}, \cdots, \pi_{\lambda}$ and invariants of orders $\leq P$;

We then obtain the information at order $P+1$ as follows

1. If necessary, we orientate the contact element of order $P$ in order for the family of frames of order $P$ to be continuous

2. We define the principal components of order $P$. To do so, we deduce, from the tables of secondary coefficients of orders $P$ and $P-1$, a system of $\nu_{P-1}-\nu_{P}$ principal components of order $P$ (equations (12)).

3. We compute their exterior derivatives (16).

4. Using formulas (10) and (11) we define coefficients of order $P$ and compute infinitesimal transformations (19) of the group $\mathcal{G}_{P}$ taken as operating on these coefficients.

5. Considering the space $W_{P}$ of these coefficients, we then determine orbits of points of $W_{P}$ under the action of $\mathcal{G}_{P}$.

6. We then trace in $W_{P}$ as simply as possible ${ }^{1}$ a manifold $w_{P}$ which intersects each orbit in one and only one point. Thus each point of $W_{P}$ has one and only one homolog on $w_{P}$. Choosing a point of $w_{P}$ amounts to choosing an orbit of $W_{P}$.

7. As frames of order $P+1$ we choose frames of order $P$ to which corresponds a point of $w_{P}$. We choose as invariants of order $P+1$ parameters which allow us to distinguish the point of $w_{P}$. In the very important special case when $\mathcal{G}_{P}$ operates transitively on $W_{P}, w_{P}$ is reduced to one point and there are no invariants of order $P+1$.

\footnotetext{
'This is all a matter of flair and insight!
} 
When point $B$ is on $w_{P}$, equations (10) and (11) yield the expressions of differentials of invariants of order $P$ and principal components of order $P$ in terms of $\pi_{1}, \cdots, \pi_{\alpha}$ and the invariants of order $P+1$. $\mathcal{G}_{P+1}$ is the subgroup of $\mathcal{G}_{P}$ which leaves point $B$ fixed. The table of secondary components of order $P+1$ is obtained from the table of order $P$ by linking differential forms $e_{q}$ with the relation (19) where we take $d b_{\alpha j}=d b_{\alpha j}^{\prime}=0$. These relations supply the principal components of order $P+1$ since it is precisely the linear combinations of forms $\omega_{i}$ which disappear for all frames of order $P+1$ with fixed origin.

We will show by the example of plane curves how the ideas developed above are applied in the case of the projective, affine and Euclidean geometry.

\section{Curves in the Euclidean Plane}

This well-known case will allow us to explain the method of moving frames. We consider Euclidean frames in the plane $\left(A, \mathbf{e}_{1}, \mathbf{e}_{2}\right)$. From what we have seen above, the equations of the most general moving frame are written

$$
\begin{aligned}
d \mathbf{A} & =\omega_{1} \mathbf{e}_{1}+\omega_{2} \mathbf{e}_{2} \\
d \mathbf{e}_{1} & =\omega_{12} \mathbf{e}_{2} \\
d \mathbf{e}_{2} & =-\omega_{12} \mathbf{e}_{1}
\end{aligned}
$$

These frames thus depend on $q=3$ parameters which is the number of parameters of the plane Euclidean group. Cartan's structure equations are very simple

$$
\begin{aligned}
d \omega_{1} & =-\omega_{2} \wedge \omega_{12} \\
d \omega_{2} & =\omega_{1} \wedge \omega_{12} \\
d \omega_{12} & =0
\end{aligned}
$$

We now consider a curve $(c)$ of class $C^{2}$ which we will assume parametrized by $t$. We will consider the set of frames whose origin is at a point of the curve $(c)$. This is a family of frames with two parameters, the parameter $t$, called the principal parameter, and the orientation $\theta$ of $e_{1}$ with respect to the horizontal, called the secondary parameter.

\subsection{Frames of order 0}

A contact element of order 0 consists of a point $A$ and all the euclidean frames having origin $A$. Frames of order 0 are characterized by the fact that point $A$ is fixed. These frames depend on only one parameter $\theta$, and we thus have, according to the notation of the previous section, $\nu_{0}=1$. Also according to that section, we must find that $q-\nu_{0}$ independent linear combinations of $\omega_{1}, \omega_{2}$ and $\omega_{12}$ disappear when we restrict them to this subclass of frames.

In effect, the three forms $\omega_{1}, \omega_{2}$ and $\omega_{12}$ are linear combinations of the forms $d t$ and $d \theta$. They are thus not independent. If we eliminate $d \theta$ within these three forms, we will obtain two two linear combinations depending only on $d t$. A linear combination of these three forms containing only $d t$ disappear for $d t=0$, i.e., when varying the frame in the family with one parameter $\theta$ relative to point $A$. Let us find these two linear combinations. 
Since frames of order 0 are characterized by the fact that the point $A$ is fixed, we have $d \mathbf{A}=\mathbf{0}$. We will call secondary coefficients, denoted by $e_{1}$ and $e_{2}$, the differential forms induced by $\omega_{1}$ and $\omega_{2}$ on this subfamily of frames, (similarly, we denote by $e_{i j}$, the restriction to the subfamily of frames under consideration of the differential form $\omega_{i j}$ ). The condition $d \mathbf{A}=\mathbf{0}$ implies that the secondary coefficients $e_{1}$ and $e_{2}$ equal zero. We have thus found our two linear combinations of the forms $\omega_{1}, \omega_{2}$ and $\omega_{12}$ which disappear along with $d t$ : they are clearly $\omega_{1}$ et $\omega_{2}$ ! We call them principal components of order 0 and, according to the previous section, we denote them by $\pi_{1}=\omega_{1}$ and $\pi_{2}=\omega_{2}$. It is useful to visualize in table form, called the table of secondary components of order 0 , the differential forms which disappear on the subfamily of frames being considered, and the relations between the others. We obtain

$$
\left[\begin{array}{cc}
0 & 0 \\
0 & e_{12} \\
-e_{12} & 0
\end{array}\right]
$$

The principal components of frames of order 0 are

$$
\begin{array}{|c|}
\hline \text { Order } 0 \\
\hline \omega_{1}, \omega_{2} \\
\hline
\end{array}
$$

\subsection{Frames of order 1}

According to the above section, we have two principal components of order 0 which are $\omega_{1}$ and $\omega_{2}$. In proposition 2.1 of the previous section, these principal components are linear combinations of $\pi_{1}$ which is equal to $\omega_{1}$. We thus have $\omega_{2}=a \omega_{1}$ and $a$ is called the coefficent of order 0 . We have to determine how this coefficient depends on secondary parameters of order 0 , i.e., on $\theta$.

In order to do this, given $\left(A, \mathbf{e}_{1}, \mathbf{e}_{2}\right)$ and $\left(B, \mathrm{f}_{1}, \mathrm{f}_{2}\right)$, two frames of order 0 , and given $\theta$ and $\bar{\theta}$, the corresponding secondary parameters, we thus have the relations

$$
\begin{array}{r}
A=B \\
\mathbf{f}_{1}=\mathbf{e}_{1} \cos (\bar{\theta}-\theta)+\mathbf{e}_{2} \sin (\bar{\theta}-\theta) \\
\mathbf{f}_{2}=-\mathbf{e}_{1} \sin (\bar{\theta}-\theta)+\mathbf{e}_{2} \cos (\bar{\theta}-\theta)
\end{array}
$$

and we have two ways of evaluating $d \mathbf{A}$

$$
d \mathbf{A}=\omega_{1} \mathbf{e}_{1}+\omega_{2} \mathbf{e}_{2}=\bar{\omega}_{1} \mathbf{f}_{1}+\bar{\omega}_{2} \mathbf{f}_{2}=\left(\bar{\omega}_{1} \cos (\bar{\theta}-\theta)-\bar{\omega}_{2} \sin (\bar{\theta}-\theta)\right) \mathbf{e}_{1}+\left(\bar{\omega}_{1} \sin (\bar{\theta}-\theta)+\bar{\omega}_{2} \cos (\bar{\theta}-\theta)\right) \mathbf{e}_{2}
$$

from which we can deduce that

$$
\begin{aligned}
& \omega_{1}=\bar{\omega}_{1} \cos (\bar{\theta}-\theta)-\bar{\omega}_{2} \sin (\bar{\theta}-\theta) \\
& \omega_{2}=\bar{\omega}_{1} \sin (\bar{\theta}-\theta)+\bar{\omega}_{2} \cos (\bar{\theta}-\theta)
\end{aligned}
$$

We thus have

$$
a=\frac{\omega_{2}}{\omega_{1}}=\frac{\sin (\bar{\theta}-\theta)+\frac{\bar{\omega}_{2}}{\bar{\omega}_{1}} \cos (\bar{\theta}-\theta)}{\cos (\bar{\theta}-\theta)-\frac{\bar{\omega}_{2}}{\bar{\omega}_{1}} \sin (\bar{\theta}-\theta)}=\frac{\sin (\bar{\theta}-\theta)+\bar{a} \cos (\bar{\theta}-\theta)}{\cos (\bar{\theta}-\theta)-\bar{a} \sin (\bar{\theta}-\theta)}
$$

Let us extract from the family of frames of order 0 attached at any point of $(c)$ the family characterized by the fact that the ratio $\frac{\bar{\omega}_{2}}{\bar{\omega}_{1}}$ has a fixed value (e.g., 0 ). This will define a subclass of the frames of order 0 , the frames of order 1 . 
Given now two frames of order 1 . From the above we have $a=\bar{a}=0$, therefore $\sin (\bar{\theta}-\theta)=0$. This shows that the family of frames of order 1 contains two elements corresponding to values $\theta$ and $\pi+\theta$ of $\bar{\theta}$. These two values give different orientations of the curve $(c)$. Since $\theta$ is fixed to within $\pi$, frames of order 1 no longer depend on any secondary parameter. We have obtained the Frenet frames of the curve.

If we consider a frame of order 1 , we see that $d \mathbf{A}=\omega_{1} \mathbf{e}_{1}$ and thus $\mathbf{e}_{1}$ is tangent to the curve at each point. According to the first structure equation, the form $\omega_{1}$ satisfies $d \omega_{1}=0$ for a frame of order 1. This is thus an exact form and we let $\omega_{1}=d s$ where $s$ is the arc length of the curve (c). The second structure equation shows that for frames of order 1 , we have $\omega_{1} \wedge \omega_{12}=0$ i.e., $\omega_{12}=\kappa \omega_{1}=\kappa d s$. This ratio $\kappa=\frac{\omega_{12}}{d s}$ is a metric invariant of the curve, its curvature at the point $A$.

The previous method is rather heavy-handed, but can be lightened and automated as follows. We consider a frame which varies while always remaining of order 0 ( its origin is fixed at a point of the curve). From the above, its principal components of order 0 are $\omega_{1}$ and $\omega_{2}$, which introduces a secondary coefficient $a$ of order 0 , such that

$$
\omega_{2}=a \omega_{1}
$$

$a$ is a function of the secondary parameter $\theta$ and of the principal parameter $t$. Let us compute the exterior differential of $\omega_{2}$, using on the one hand the previous expression, and on the other hand the structure equations (8)

$$
d \omega_{2}=d a \wedge \omega_{1}+a d \omega_{1}=\omega_{1} \wedge \omega_{12}
$$

Let us use the fact that $d \omega_{1}=-\omega_{2} \wedge \omega_{12}=-a \omega_{1} \wedge \omega_{12}$. We obtain

$$
\omega_{1} \wedge\left(d a+\omega_{12}\left(1+a^{2}\right)\right)=0
$$

We thus have $d a+\omega_{12}\left(1+a^{2}\right)=\alpha \omega_{1}$, and since $\omega_{1}=0$ for frames of order 0 , we finally obtain

$$
d a=-\left(a^{2}+1\right) e_{12}
$$

The group $\mathcal{G}_{0}$ operates transitively on $a$ and we can choose a subset such that $a=0$ from the class of frames of order 0 . These frames are called frames of order 1 . They therefore satisfy the condition $\omega_{2}=0$. The vector $e_{1}$ is thus tangent to the curve. According to the previous equation, this implies $e_{12}=0$. Frames of order 1 do not depend on any secondary parameter and we thus have $\nu_{1}=0$. From the previous section, an infinitesimal translation of the frame of order 1 zeros $q-\nu_{0}=2$ linear combinations of $\omega_{1}, \omega_{2}, \omega_{12}\left(\omega_{1}\right.$ and $\left.\omega_{2}\right)$ and $\nu_{0}-\nu_{1}=1$ new one, which is consequently $\omega_{12}$.

Thus the table of secondary components of order 1 is

$$
\left[\begin{array}{ll}
0 & 0 \\
0 & 0 \\
0 & 0
\end{array}\right]
$$

The principal components of frames of order 1 are

\begin{tabular}{|c|c|}
\hline Order 0 & Order 1 \\
\hline$\omega_{1}, \omega_{2}(=0)$ & $\omega_{12}$ \\
\hline
\end{tabular}


We find here again that the secondary coefficient of order 1 , the ratio $\frac{w_{12}}{d_{s}}$, is an invariant of order 2 which we identify as the curvature $\kappa$. We have the classical Frenet formulas

$$
\begin{aligned}
& \frac{d \mathrm{~A}}{d s}=\mathrm{e}_{1} \\
& \frac{d \mathrm{e}_{1}}{d s}=\kappa \mathrm{e}_{2} \\
& \frac{d \mathrm{e}_{2}}{d s}=-\kappa \mathrm{e}_{1}
\end{aligned}
$$

\subsection{Application to the evolution of curves}

We are now going to consider the case of a family of curves. In order to deal with this problem, we change our notation slightly and consider a closed embedded smooth curve (i.e. a curve with no self-intersections). We denote by $p$ the principal parameter. The curve can be considered as a mapping $\mathrm{A}: \mathcal{S}^{1} \rightarrow R^{2}$ where $\mathcal{S}^{1}$ is the unit circle. We now consider smooth embedded plane curves deforming in time. Let $\mathbf{A}(p, t): \mathcal{S}^{1} \times R \rightarrow R^{2}$ be a family of such curves where $p$ parametrizes the curve and $t$ represents the time. Let $s$ be the euclidean arc-length along a curve of the family, a function of $p$ and $t$. We now propose to study the following evolution equation:

$$
\mathbf{A}_{t}=\mathbf{A}_{s s}
$$

in which the partial with respect to $t$ is taken at $p$ constant and the partials with respect to $s$ are taken at $t$ constant. This equation can be thought of as a heat equation (because of the formal similarity with the usual heat equation) which is intrinsic to the curve. It has been studied by Gage and Hamilton $[8,9,10]$ who proved that a planar convex embedded curve converges to a round point when evolving according to (24) and Grayson [11] who proved that a planar embedded nonconvex curve converges first to a convex one and then to a round point.

Since a curve in the euclidean plane is defined up to a rigid transformation by its arclength and curvature, it is natural to establish how they evolve in time when the curve changes according to (24). The key is of course to use the Frenet equations (23).

We first derive a general result which we will use also in later sections. It is related to the fact that the operators of partial derivative with respect to $t$ (at $p$ constant) and with respect to $s$ (at $t$ constant) do not commute since $s$ is a function of $p$ and $t$. Let then $g$ equal $\frac{d s}{d p}$, it is easy to show that the Lie bracket $\left[\frac{\partial}{\partial t}, \frac{\partial}{\partial s}\right]$ is equal to

$$
\left[\frac{\partial}{\partial t}, \frac{\partial}{\partial s}\right]=\frac{\partial^{2}}{\partial t \partial s}-\frac{\partial^{2}}{\partial s \partial t}=-\frac{g_{t}}{g} \frac{\partial}{\partial s}
$$

Applying this formula twice, we obtain the following expressions which we will find useful later:

$$
\frac{\partial}{\partial t} \frac{\partial^{2}}{\partial s^{2}}=-\left[\frac{g_{t}}{g}\right]_{8} \frac{\partial}{\partial s}-2 \frac{g_{t}}{g} \frac{\partial^{2}}{\partial s^{2}}+\frac{\partial^{2}}{\partial s^{2}} \frac{\partial}{\partial t}
$$

and

$$
\frac{\partial}{\partial t} \frac{\partial^{3}}{\partial s^{3}}=-\left[\frac{g_{t}}{g}\right]_{\rho^{2}} \frac{\partial}{\partial s}-3\left[\frac{g_{t}}{g}\right]_{0} \frac{\partial^{2}}{\partial s^{2}}-3 \frac{g_{t}}{g} \frac{\partial^{3}}{\partial s^{3}}+\frac{\partial^{3}}{\partial s^{3}} \frac{\partial}{\partial t}
$$


Similarly, we will need the following expressions of the higher order derivatives of $\mathbf{A}$ with respect to the arclength $s$ which we obtain from the Frenet formulae:

$$
\begin{aligned}
& \mathbf{A}_{s^{3}}=-\kappa^{2} \mathbf{e}_{1}+\kappa_{s} \mathbf{e}_{2} \\
& \mathbf{A}_{s^{4}}=-3 \kappa_{s} \kappa \mathbf{e}_{1}+\left(\kappa_{s s}-\kappa^{3}\right) \mathbf{e}_{2} \\
& \mathbf{A}_{s^{5}}=\left(\kappa^{4}-3 \kappa_{s}^{2}-4 \kappa_{s s} \kappa\right) \mathbf{e}_{1}+\left(\kappa_{s^{3}}-6 \kappa_{s} \kappa^{2}\right) \mathbf{e}_{2}
\end{aligned}
$$

\subsubsection{Evolution of arc-length}

It is now easy to characterize the evolution of arc-length. We use the relation

$$
\mathbf{e}_{1} \cdot \mathbf{e}_{1}=1
$$

which can be rewritten as

$$
A_{s} \cdot A_{s}=1
$$

and take its derivative with respect to $t$ :

$$
\mathbf{A}_{\text {to }} \cdot \mathbf{A}_{s}=0
$$

We then use equations (25) and (24) to rewrite

$$
\mathbf{A}_{t s}=-\frac{g_{t}}{g} \mathbf{A}_{s}+\mathbf{A}_{s}
$$

Using equation (28) we finally get

$$
g_{t}=-\kappa^{2} g
$$

\subsubsection{Evolution of curvature}

The principle of the method is to use a differential equation that is satisfied by each curve of the family. Using the Frenet equations and equation (28) we obtain

$$
\kappa \mathbf{A}_{s^{3}}-\kappa_{s} \mathbf{A}_{s^{2}}+\kappa^{3} \mathbf{A}_{s}=0
$$

We take the derivative of this equation with respect to $t$ :

$$
\kappa_{t} \mathbf{A}_{s^{3}}+\kappa \mathbf{A}_{t s^{3}}-\kappa_{t s} \mathbf{A}_{s^{2}}-\kappa_{s} \mathbf{A}_{t s^{2}}+3 \kappa_{t} \kappa^{2} \mathbf{A}_{s}+\kappa^{3} \mathbf{A}_{t s}=0
$$

We then use equations (25)-(27), the Frenet equations and equations (28)-(30) to obtain the equation

$$
P\left(\kappa, \kappa_{t}, \kappa_{s}, \kappa_{s^{2}}\right) \mathbf{e}_{1}+Q\left(\kappa, \kappa_{t}, \kappa_{s}, \kappa_{s^{2}}, \kappa_{s^{3}}, \kappa_{t s}\right) \mathbf{e}_{2}=0
$$

This implies $P=Q=0$. The first equation yields the sought for evolution equation:

$$
\kappa_{t}=\kappa_{s s}+\kappa^{2}
$$

We then replace $\kappa_{t s}$ by $-\frac{g_{t}}{g} \kappa_{s}+\kappa_{s t}$ in $Q$ thanks to equation (25), use equation (32) and find that $Q$ is identically 0. Equation (32) is an example of a special kind of partial differential equation called a reaction diffusion equation. These equations have been studied quite extensively in mathematics (see for example [22]). In fact we have here a system of two coupled pde's (equations (31) and (32)) which must be studied as such. 


\section{Affine unimodal bidimensional geometry}

We will consider the affine frames of the plane $\left(A, \mathbf{e}_{1}, \mathbf{e}_{2}\right)$. In section 2 , the equations of the most general moving frame are written

$$
\begin{aligned}
d \mathbf{A} & =\omega_{1} \mathbf{e}_{1}+\omega_{2} \mathbf{e}_{2} \\
d \mathbf{e}_{1} & =\omega_{11} \mathbf{e}_{1}+\omega_{12} \mathbf{e}_{2} \\
d \mathbf{e}_{2} & =\omega_{21} \mathbf{e}_{1}-\omega_{11} \mathbf{e}_{2}
\end{aligned}
$$

Thus these frames depend on $q=5$ parameters which is the number of parameters of the plane unimodular affine group.

\subsection{Frames of order 0}

A contact element of order 0 consists of a point $A$ and all the affine frames of origin $A$. Frames of order 0 are characterized by the fact that the point $A$ is fixed, thus $d \mathbf{A}=0$, which implies that the secondary coefficients $e_{1}$ and $e_{2}$ equal $0: q-\nu_{0}=2$. Thus frames of order 0 depend on $\nu_{0}=3$ parameters. We have two principal components of order $0, \pi_{1}=\omega_{1}$ and $\pi_{2}=\omega_{2}$. As mentioned above, it is convenient to set the differential of the principal parameter which defines the position of a point of the curve equal to the first principal component, i.e., to $\omega_{1}$. The table of secondary components of order 0 is given by

$$
\left[\begin{array}{cc}
0 & 0 \\
e_{11} & e_{12} \\
e_{21} & -e_{11}
\end{array}\right]
$$

The principal components of frames of order 0 are

$$
\begin{array}{|c|}
\hline \text { Order } 0 \\
\hline \omega_{1}, \omega_{2} \\
\hline
\end{array}
$$

\subsection{Frames of order 1}

Given a frame which varies while always remaining of order 0 (its origin is fixed at one point of the curve). According to the above, its principal components of order 0 are linear combinations of $\pi_{1}$, which introduces a coefficient $a$ of order 0 , such that $\pi_{2}=a \pi_{1}$, i.e.,

$$
\omega_{2}=a \omega_{1}
$$

Let us compute the exterior differential of $\omega_{2}$ using on the one hand the previous expression, and on the other hand the structure equations (8)

$$
d \omega_{2}=d a \wedge \omega_{1}+a d \omega_{1}
$$

Using the facts that $d \omega_{1}=\omega_{1} \wedge \omega_{11}+\omega_{2} \wedge \omega_{21}=\omega_{1} \wedge\left(\omega_{11}+a \omega_{21}\right)$ and $d \omega_{2}=\omega_{1} \wedge \omega_{12}+\omega_{2} \wedge \omega_{22}=$ $\omega_{1} \wedge\left(\omega_{12}+a \omega_{22}\right)$, we obtain

$$
\omega_{1} \wedge\left(d a+\omega_{12}+a\left(\omega_{22}-\omega_{11}\right)-a^{2} \omega_{21}\right)=0
$$


Therefore we have $d a+\omega_{12}+a\left(\omega_{22}-\omega_{11}\right)-a^{2} \omega_{21}=\alpha \omega_{1}$, and as $\omega_{1}=0$ for frames of order 0 , we obtain

$$
d a=-e_{12}-a\left(e_{22}-e_{11}\right)+a^{2} e_{21}
$$

The group $\mathcal{G}_{0}$ thus operates transitively on $a$. We can choose a subset such that $a=0$ from the class of frames of order 0 . These frames are called frames of order 1 . They thus satisfy the condition $\omega_{2}=0$. The vector $e_{1}$ is therefore tangent to the curve. According to the equation shown above, this implies $e_{12}=0$.

We thus have a principal component $\omega_{12}$ of order 1 . Thus $\nu_{0}-\nu_{1}=1$ and frames of order 1 depend on $\nu_{1}=2$ secondary parameters. The table of secondary components of order 1 is given by

$$
\left[\begin{array}{cc}
0 & 0 \\
e_{11} & 0 \\
e_{21} & -e_{11}
\end{array}\right]
$$

The principal components of frames of order 1 are

\begin{tabular}{|c|c|}
\hline Order 0 & Order 1 \\
\hline$\omega_{1}, \omega_{2}(=0)$ & $\omega_{12}$ \\
\hline
\end{tabular}

\subsection{Frames of order 2}

Given a frame which varies while always remaining of order 1 (its origin is fixed at a point of the curve and the vector $e_{1}$ is tangent to the curve). According to the above, its principal components of order 1 are linear combinations of $\pi_{1}$, which introduces a coefficient $a$ of order 1 , such that $\omega_{12}=a \omega_{1}$. Let us compute the exterior differential of $\omega_{12}$ using on the one hand the previous expression, and on the other hand the structure equations (8)

$$
d \omega_{12}=d a \wedge \omega_{1}+a d \omega_{1}
$$

Since $\omega_{2}=0$, we have $d \omega_{1}=\omega_{1} \wedge \omega_{11}$. Moreover, $d \omega_{12}=\omega_{11} \wedge \omega_{12}+\omega_{12} \wedge \omega_{22}=a \omega_{1} \wedge\left(\omega_{22}-\omega_{11}\right)$ and we can deduce that

$$
d a+3 a w_{11}=\alpha \omega_{1}
$$

Since we took into account the relation $\omega_{22}=-\omega_{11}$ and since $\omega_{1}=0$, we have

$$
d a=-3 a e_{11}
$$

The group $\mathcal{G}_{1}$ operates transitively on $a$. We can choose a subset such that $a=1$ from the class of frames of order 1 . These frames are called frames of order 2 . They satisfy the condition $\omega_{12}=\omega_{1}$ and the coefficient $e_{11}$ equals zero. We thus have a principal component $\omega_{11}$ of order 2 , and $\nu_{1}-\nu_{2}=1$. Frames of order 2 depend on $\nu_{2}=1$ parameter. The table of secondary components of order 2 is

$$
\left[\begin{array}{cc}
0 & 0 \\
0 & 0 \\
e_{21} & 0
\end{array}\right]
$$

The principal components of frames of order 2 are

\begin{tabular}{|c|c|c|}
\hline Order 0 & Order 1 & Order 2 \\
\hline$\omega_{1}, \omega_{2}(=0)$ & $\omega_{12}\left(=\omega_{1}\right)$ & $\omega_{11}$ \\
\hline
\end{tabular}




\subsection{Frames of order 3}

Given a frame which varies while always remaining of order 2. According to the above, its principal components of order 2 are linear combinations of $\pi_{1}$, which introduces a coefficient $a$ of order 2 , such that $\omega_{11}=a \omega_{1}$. Let us compute the exterior differential of $\omega_{11}$ using on the one hand the previous expression, and on the other hand the structure equations (8)

$$
d \omega_{11}=d a \wedge \omega_{1}+a d \omega_{1}
$$

We still have $d \omega_{1}=\omega_{1} \wedge \omega_{11}$ and $d \omega_{11}=\omega_{12} \wedge \omega_{21}=\omega_{1} \wedge \omega_{21}$. Hence we can easily deduce

$$
d a=-e_{21}
$$

Thus the group $\mathcal{G}_{2}$ operates transitively on $a$. Its action consists in adding any constant to $a$. We can choose a subset such that $a=0$ from the class of frames of order 2 . These frames are called frames of order 3. They satisfy the condition $\omega_{11}=0$ and the coefficient $e_{21}$ equals zero. We thus have a principal component $\omega_{21}$ of order 2 and $\nu_{2}-\nu_{3}=1$. The frames of order 3 do not depend on any parameter. The principal components of frames of order 3 are

\begin{tabular}{|c|c|c|c|}
\hline Order 0 & Order 1 & Order 2 & Order 3 \\
\hline$\omega_{1}, \omega_{2}(=0)$ & $\omega_{12}\left(=\omega_{1}\right)$ & $\omega_{11}(=0)$ & $\omega_{21}$ \\
\hline
\end{tabular}

The form $\omega_{1}$ satisfies $d \omega_{1}=0$ and is therefore exact. We let

$$
\omega_{1}=d \sigma
$$

where $\sigma$ is called the affine arc length parameter. We have an invariant of order 4 , the ratio $\frac{\omega_{22}}{d \sigma}$, which is the affine curvature $k$. Frames of order 3 are Frenet frames and we have the following equations

$$
\begin{aligned}
& \frac{d \mathbf{A}}{d \sigma}=\mathbf{e}_{1} \\
& \frac{d \mathbf{e}_{1}}{d \sigma}=\mathbf{e}_{2} \\
& \frac{d \mathbf{e}_{2}}{d \sigma}=k \mathbf{e}_{1}
\end{aligned}
$$

\subsection{Analytic determination of arc length and affine curvature}

Supposing the curve parametrized by any parameter $t$ and using the Frenet formulas, we have

$$
\begin{array}{r}
\frac{d \mathbf{A}}{d t}=\frac{d \mathbf{A}}{d \sigma} \frac{d \sigma}{d t}=\mathrm{e}_{1} \frac{d \sigma}{d t} \\
\frac{d^{2} \mathbf{A}}{d t^{2}}=\mathrm{e}_{1} \frac{d^{2} \sigma}{d t^{2}}+\mathrm{e}_{2}\left(\frac{d \sigma}{d t}\right)^{2}
\end{array}
$$

Taking the vector products and with $e_{1} \times e_{2}=1$, we obtain

$$
\frac{d \sigma}{d t}=\left(\frac{d \mathbf{A}}{d t} \times \frac{d^{2} \mathbf{A}}{d t^{2}}\right)^{1 / 3}
$$


This equation will allow us to compute the affine arc length parametrization from any parametization of the curve. Pushing the derivative further will enable us to compute the affine curvature. In effect

$$
\frac{d^{3} \mathbf{A}}{d t^{3}}=\left(k\left(\frac{d \sigma}{d t}\right)^{3}+\frac{d^{3} \sigma}{d t^{3}}\right) \mathbf{e}_{1}+3 \frac{d \sigma}{d t} \frac{d^{2} \sigma}{d t^{2}} \mathbf{e}_{2}
$$

Taking the vector product with $\frac{d^{2} \mathrm{~A}}{d t^{2}}$ and extracting $k$ yields

$$
k=-\frac{\frac{d^{3} \sigma}{d t^{3}}}{\left(\frac{d \sigma}{d t}\right)^{3}}+3 \frac{\left(\frac{d^{2} \sigma}{d t^{2}}\right)^{2}}{\left(\frac{d \sigma}{d t}\right)^{4}}-\frac{1}{\left(\frac{d \sigma}{d t}\right)^{5}} \frac{d^{2} \mathbf{A}}{d t^{2}} \times \frac{d^{3} \mathbf{A}}{d t^{3}}
$$

This expression is true for any parameter $t$.In the case where $t=s$, the Euclidean arc length, we have

$$
\begin{array}{rcl}
\frac{d \mathbf{A}}{d s}=\mathrm{t} & \frac{d^{2} \mathbf{A}}{d s^{2}}=\frac{\mathbf{n}}{R} & \frac{d^{3} \mathbf{A}}{d s^{3}}=-\frac{\mathbf{t}}{R^{2}}-\frac{\frac{d R}{d s}}{R^{2}} \mathbf{n} \\
\frac{d \sigma}{d s}=R^{-\frac{1}{3}} & \frac{d^{2} \sigma}{d s^{2}}=-\frac{1}{3} \frac{d R}{d s} R^{-\frac{3}{4}} & \frac{d^{3} \sigma}{d s^{3}}=-\frac{1}{3} \frac{d^{2} R}{d s^{2}} R^{-\frac{3}{4}}+\frac{4}{9}\left(\frac{d R}{d s}\right)^{2} R^{-\frac{7}{3}}
\end{array}
$$

By replacing these terms with their values in equation (35), we obtain

$$
k=-R^{-\frac{4}{3}}+\frac{1}{3} \frac{d^{2} R}{d s^{2}} R^{-\frac{1}{3}}-\frac{1}{9}\left(\frac{d R}{d s}\right)^{2} R^{-\frac{1}{3}}
$$

We recognize the common term $\frac{1}{2} \frac{d^{2} R^{\frac{2}{3}}}{d s^{2}}$ in the last two terms of the right-hand side, and we obtain this handsome formula

$$
k=-R^{-\frac{1}{3}}+\frac{1}{2} \frac{d^{2} R^{\frac{2}{3}}}{d s^{2}}
$$

Note that we have obtained the relation between the affine arc length parameter $\sigma$ and the Euclidean arc length parameter $s$

$$
\frac{d \sigma}{d s}=\kappa^{\frac{1}{3}}
$$

where $\kappa$ is the Euclidean curvature. This, naturally, also yields the Euclidean norm of the affine tangent $\mathbf{e}_{1}$

$$
\mathbf{e}_{1}=\frac{d \mathbf{A}}{d \sigma}=\frac{d \mathbf{A}}{d s} \frac{d s}{d \sigma}=\mathbf{t} R^{\frac{1}{3}}
$$

so that

$$
\left|\mathrm{e}_{1}\right|=|R|^{\frac{1}{3}}=|\kappa|^{-\frac{1}{3}}
$$

We can continue and compute the components of the affine normal $e_{2}$ in the basis $(t, n)$

$$
\mathrm{e}_{2}=\frac{d \mathrm{e}_{1}}{d \sigma}=\frac{d R^{\frac{1}{3}}}{d s} \kappa^{-\frac{1}{3}} \mathrm{t}+\kappa^{\frac{1}{3}} \mathbf{n}
$$

The component of the affine normal on the Euclidean normal is therefore equal to $\kappa^{\frac{2}{3}}$. This remark turned out to be of great importance in the affine "scale-space" analysis $[19,18,20,1]$. 


\subsection{Geometric interpretation}

We take two points $A$ and $B$, and two line directions $d$ and $d^{\prime}$ represented by two vectors that we denote $\mathbf{A}^{\prime}$ and $\mathbf{B}^{\prime}$. These four elements determine a triangle $A B A_{01}$, where $A_{01}$ is obtained as the intersection of the lines passing through $A$ parallel to $\mathbf{A}^{\prime}$ and through $B$ parallel to $\mathbf{B}^{\prime}$. It is easy to show that the area $S$ of the triangle $A B A_{01}$ is given by the following formula

$$
S=\frac{1}{2} \frac{\left(\mathbf{A}^{\prime} \times \mathbf{B A}\right) \cdot\left(\mathbf{B A} \times \mathbf{B}^{\prime}\right)}{\mathbf{A}^{\prime} \times \mathbf{B}^{\prime}}
$$

The quadruple $A, B, \mathbf{A}^{\prime}$ and $\mathbf{B}^{\prime}$ also determines in a unique way a parabola passing through $A$ and $B$, and tangent to $\left\langle A, A_{01}\right\rangle$ and $\left\langle B, A_{01}\right\rangle$. The equation of this parabola is written

$$
\mathbf{A}(t)=\mathbf{A}_{0}+t \mathbf{A}_{0}^{\prime}+\frac{t^{2}}{2} \mathbf{A}_{0}^{\prime \prime}
$$

with

$$
\begin{array}{rr}
\mathbf{A}_{0}=\mathbf{A}\left(t_{0}\right)=\mathbf{A}(0)=\mathbf{A} & \mathbf{A}_{1}=\mathbf{A}\left(t_{1}\right)=\mathbf{B} \\
\left.\frac{d \mathbf{A}}{d t}\right|_{t=t_{0}} \times \mathbf{A}^{\prime}=\mathbf{0} & \left.\frac{d \mathbf{A}}{d t}\right|_{t=t_{1}} \times \mathbf{B}^{\prime}=\mathbf{0}
\end{array}
$$

Let us compute the area of the triangle $A B A_{01}$ in terms of $t_{1}$. We use (39) to write

$$
S\left(t_{1}\right)=\frac{1}{2} \frac{\left(\mathbf{A}_{0}^{\prime} \times \mathbf{A}_{0} \mathbf{A}_{1}\right) \cdot\left(\mathbf{A}_{0} \mathbf{A}_{1} \times \mathbf{A}_{1}^{\prime}\right)}{\mathbf{A}_{0}^{\prime} \times \mathbf{A}_{1}^{\prime}}
$$

We replace $\mathbf{A}_{0} \mathbf{A}_{1}$ in the previous equation by $t_{1} \mathbf{A}_{0}^{\prime}+\frac{t 1^{2}}{2} \mathbf{A}_{0}^{\prime \prime}$ and $\mathbf{A}_{1}^{\prime}$ by $\mathbf{A}_{0}^{\prime}+t_{1} \mathbf{A}_{0}^{\prime \prime}$, and we obtain

$$
S\left(t_{1}\right)=\frac{1}{8} t_{1}^{3} \mathbf{A}_{0}^{\prime} \times \mathbf{A}_{0}^{\prime \prime}
$$

The area of the triangle defined by two points of the parabola at parameters $t_{1}$ and $t_{2}$ is thus given by the formula

$$
S\left(t_{1}, t_{2}\right)=\frac{1}{8}\left(t_{2}-t_{1}\right)^{3} \mathbf{A}_{0}^{\prime} \times \mathbf{A}_{0}^{\prime \prime}
$$

From which we deduce the following addition law

$$
S\left(t_{0}, t_{1}\right)^{\frac{2}{3}}+S\left(t_{1}, t_{2}\right)^{\frac{1}{3}}=S\left(t_{0}, t_{2}\right)^{\frac{1}{3}}
$$

which was already known to Möbius (see figure 1).

Let us demonstrate that this law is nothing but the addition law of affine arc length along the parabola. In effect, according to the equation (34), we have

$$
\frac{d \sigma}{d t}=\left(\frac{d \mathbf{A}}{d t} \times \frac{d^{2} \mathbf{A}}{d t^{2}}\right)^{1 / 3}
$$

so that, according to $(40)$

$$
\frac{d \sigma}{d t}=\left(\mathbf{A}_{0}^{\prime} \times \mathbf{A}_{0}^{\prime \prime}\right)^{\frac{1}{3}}
$$

We can conclude that $\sigma(t)=2 S(t)^{\frac{1}{3}}$, which gives the addition formula. 


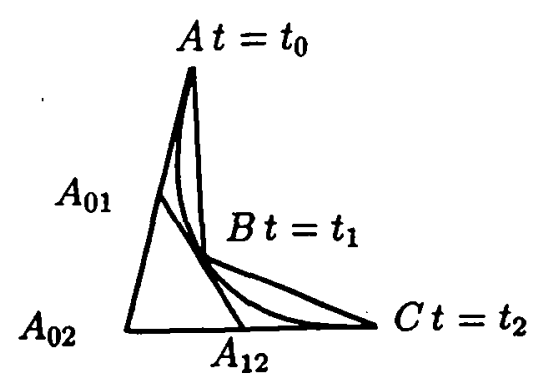

Figure 1: We have the curious relation Area $\left(A B A_{01}\right)^{\frac{1}{3}}+\operatorname{Area}\left(B C A_{12}\right)^{\frac{1}{3}}=\operatorname{Area}\left(A C A_{02}\right)^{\frac{1}{3}}$

\subsection{The affine group}

It is interesting to make a table of the dimensions of the various elements involved with affine curves.

\begin{tabular}{|c|c|c|c|c|c|c|}
\hline $\mathbf{A}$ & $\sigma$ & $\mathbf{A}^{\prime}$ & $\mathbf{A}^{\prime \prime}$ & $\mathbf{A}^{\prime \prime}$ & $k$ & $k^{\prime}$ \\
\hline 1 & $\frac{2}{3}$ & $\frac{1}{3}$ & $-\frac{1}{3}$ & -1 & $-\frac{4}{3}$ & -2 \\
\hline
\end{tabular}

This table is used in the following manner. If we multiply the lengths by $\lambda$, then the arc length parameter $\sigma$ is multiplied by $\lambda^{\frac{2}{3}}$, etc.

We can see, in particular, that the integral

$$
\int|k|^{\frac{1}{2}} d \sigma
$$

is invariant by the affine group.

\subsection{Application to the evolution of curves}

Similarly to the euclidean case (see section 3.3), we are now going to consider the case of a family of curves. We use the same notations as in this section and consider smooth embedded plane curves deforming in time. Let $\mathbf{A}(p, t): \mathcal{S}^{1} \times R \rightarrow R^{2}$ be a family of such curves where $p$ parametrizes the curve and $t$ represents the time. Let $\sigma$ be the affine arc length along a curve of the family, a function of $p$ and $t$. We now propose to study the following evolution equation:

$$
\mathbf{A}_{t}=\mathbf{A}_{\sigma \sigma}
$$

in which the partial with respect to $t$ is taken at $p$ constant and the partials with respect to $\sigma$ are taken at $t$ constant. This equation, as (24), can be thought of as a heat equation (because of the formal similarity with the usual heat equation) which is intrinsic to the curve. It has been studied by Sapiro and Tannenbaum $[19,18,21,20]$ who proved that a planar convex embedded curve converges to an elliptic point when evolving according to (41) and that a planar embedded nonconvex curve converges first to a convex one and then to an elliptic point.

Since a curve in the affine plane is defined up to an affine transformation by its arc length and curvature, it is natural to establish how they evolve in time when the curve changes according to (41). The key is of course to use the Frenet equations (33). 
We use the equations (25), (26), (27) which are unchanged in the affine framework. We will also need the following expressions of the higher order derivatives of $\mathbf{A}$ with respect to the arc length $\sigma$ which we obtain from the Frenet formulae:

$$
\begin{aligned}
& \mathbf{A}_{\sigma^{3}}=k \mathbf{e}_{1} \\
& \mathbf{A}_{\sigma^{4}}=k_{\sigma} \mathbf{e}_{1}+k \mathbf{e}_{2} \\
& \mathbf{A}_{\sigma^{5}}=\left(k^{2}+k_{\sigma}^{2}\right) \mathbf{e}_{1}+2 \kappa_{\sigma} \mathbf{e}_{2}
\end{aligned}
$$

\subsubsection{Evolution of affine arc length}

It is now easy to characterize the evolution of arc length. We let $g=\frac{d \sigma}{d p}$ and use the relation

$$
\mathbf{e}_{1} \wedge \mathbf{e}_{2}=1
$$

which can be rewritten as

$$
\mathbf{A}_{\sigma} \wedge \mathbf{A}_{\sigma \sigma}=1
$$

and take its derivative with respect to $t$ :

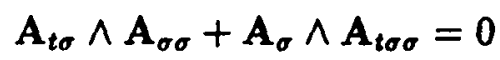

We then use equations (25) and (41) to rewrite

$$
\mathbf{A}_{t \sigma}=-\frac{g_{t}}{g} \mathbf{A}_{\sigma}+\mathbf{A}_{\sigma}
$$

Using equation (42) we obtain

$$
\mathbf{A}_{t \sigma} \wedge \mathbf{A}_{\sigma \sigma}=\left(k-\frac{g_{t}}{g}\right) \mathbf{A}_{\sigma} \wedge \mathbf{A}_{\sigma \sigma}=k-\frac{g_{t}}{g}
$$

Similarly, using equations (26) and (43), we obtain

$$
\mathbf{A}_{\sigma} \wedge \mathbf{A}_{t \sigma \sigma}=\left(k-2 \frac{g_{t}}{g}\right) \mathbf{A}_{\sigma} \wedge \mathbf{A}_{\sigma \sigma}=k-2 \frac{g_{t}}{g}
$$

and finally

$$
g_{t}=-\frac{2}{3} k g
$$

which is the affine analog of (31).

\subsubsection{Evolution of affine curvature}

The principle of the method is to use a differential equation that is satisfied by each curve of the family. Using the Frenet equations and equation (42) we obtain

$$
\mathbf{A}_{\sigma^{3}}-k \mathbf{A}_{\sigma}=0
$$

We take the derivative of this equation with respect to $t$ :

$$
\mathbf{A}_{t \sigma^{3}}-k_{t} \mathbf{A}_{\sigma}-k \mathbf{A}_{t \sigma}=0
$$


We then use equations (25)-(27), the Frenet equations, equations (42)-(45) to obtain the equation

$$
\left(\frac{1}{3} k_{\sigma \sigma}-\frac{4}{3} k^{2}-k_{t}\right) \mathbf{e}_{1}=0
$$

This yields the sought-for evolution equation:

$$
k_{t}=\frac{1}{3} k_{\sigma \sigma}-\frac{4}{3} k^{2}
$$

which is the affine analog of (32).

\subsubsection{Evolution of euclidean curvature}

It is interesting to look at the temporal evolution of the euclidean curvature when the curve evolves according to (41). In order to do this, we use equation (38) which says:

$$
\mathbf{A}_{\sigma} \cdot \mathbf{A}_{\sigma}=\kappa^{-\frac{2}{3}}
$$

where $\kappa$ is the euclidean curvature. If we take the derivative of this equation with respect to time, we obtain

$$
\mathbf{A}_{t \sigma} \cdot \mathbf{A}_{\sigma}=-\frac{1}{3} \kappa_{t} \kappa^{-\frac{8}{3}}
$$

From previous computation, we know that $\mathbf{A}_{t \sigma}=\frac{1}{3} k \mathbf{A}_{\sigma}$, where $k$ is the affine curvature. Thus we obtain

$$
\kappa_{t}=-k \kappa
$$

This equation is interesting because it says that we can save one order of derivation by using the scalespace defined by the curve evolution (41). Indeed, according to equation (36), the affine curvature can be obtained from a second order derivative of the euclidean curvature with respect to euclidean arc-length. But, according to equation (47), we can obtain the affine curvature from a first order derivative of the euclidean curvature with respect to time.

\section{Plane projective geometry}

We will now consider projective frames in the plane $\left(A, A_{1}, A_{2}\right)$. As in $\S 2.2$, the equations of the most general moving frame are

$$
\begin{aligned}
d \mathbf{A} & =\omega_{00} \mathbf{A}+\omega_{01} \mathbf{A}_{1}+\omega_{02} \mathbf{A}_{2} \\
d \mathbf{A}_{1} & =\omega_{10} \mathbf{A}+\omega_{11} \mathbf{A}_{1}+\omega_{12} \mathbf{A}_{2} \\
d \mathbf{A}_{2} & =\omega_{20} \mathbf{A}+\omega_{21} \mathbf{A}_{1}+\omega_{22} \mathbf{A}_{2}
\end{aligned}
$$

with the relation

$$
\omega_{00}+\omega_{11}+\omega_{22}=0
$$

The most general moving frame thus depends on $q=8$ parameters, which is the number of parameters of the plane projective group. 
The moving frame method can be applied in a straightforward fashion to this case and we do not give the details here. The interested reader is referred to [4]. We find that, in order to get to the Frenet frame, we have to consider frames of order up to 6.

The differential $\omega_{01}$ is exact. Let $\omega_{01}=d \sigma$ where $\sigma$ is called the projective arc length parameter. The secondary coefficient $\frac{\omega_{10}}{\omega_{01}}$ of order 6 is an invariant of order 7 . We write

$$
\frac{\omega_{10}}{\omega_{01}}=\frac{\omega_{10}}{d \sigma}=-k
$$

where $k$ is the projective curvature. Frames of order 6 are the Frenet frames and we have the following equations

$$
\begin{aligned}
\frac{d \mathbf{A}}{d \sigma} & =\mathbf{A}_{1} \\
\frac{d \mathbf{A}_{1}}{d \sigma} & =-k \mathbf{A}+\mathbf{A}_{\mathbf{2}} \\
\frac{d \mathbf{A}_{2}}{d \sigma} & =-\mathbf{A}-k \mathbf{A}_{1}
\end{aligned}
$$

\subsection{Geometric interpretation}

We now give a useful geometric interpretation of the projective arclength. Let us consider two points $A$ and $B$ on a curve $(c)$, the tangent $T_{A}$ to $(c)$ at $A$, the tangent $T_{B}$ to $(c)$ at $B$ and the chord $\langle A, B\rangle$. We then consider the pencil of conics going through the points $A$ and $B$ and tangent there to $T_{A}$ and $T_{B}$. The equation of each conic in this pencil can be written as

$$
T_{A} T_{B}+\lambda\langle A, B\rangle^{2}
$$

where $\lambda$ varies between $-\infty$ and $\infty$. Indeed the degenerate conic $\left(c_{1}\right)$ composed of the two lines $T_{A}$ and $T_{B}$ of equation $T_{A} T_{B}=0$ and the degenerate conic $\left(c_{2}\right)$ composed of the double line $\langle A, B\rangle$ of equation $\langle A, B\rangle^{2}=0$ both belong to the pencil, the first one being obtained for the value $\lambda_{1}=0$ of $\lambda$, the second for the value $\lambda_{2}=\infty$. Among the conics of this pencil, there is one $\left(c_{A}\right)$ with a contact of order 3 with the curve $(c)$ at $A$ and one, $\left(c_{B}\right)$ with a contact of order 3 with the curve (c) at $B$. Let $\lambda_{A}$ (resp. $\lambda_{B}$ ) the corresponding values of the projective parameter $\lambda$. We consider the cross-ratio of the four conics $\left(\left(c_{A}\right),\left(c_{B}\right),\left(c_{1}\right),\left(c_{2}\right)\right)$. It is a projective invariant equal to the ratio $\lambda_{A} / \lambda_{B}$. We relate it to the projective arc-length $\sigma_{B}-\sigma_{A}$ between the two points $A$ and $B$ on the curve $(c)$.

Let us consider the Frenet frame $\left(A, A_{1}, A_{2}\right)$ at $A$. We represent each each point $M$ of the projective plane by its coordinates $x, y$ in this coordinate system, i.e.:

$$
\mathbf{M}=\mathbf{A}+x \mathbf{A}_{1}+y \mathbf{A}_{2}
$$

Finally, we choose $A$ as the origin of arclength $\sigma$. Let $X(\sigma), Y(\sigma)$ be the coordinates of $B$ and let us compute the equations of $T_{A}, T_{B},\langle A, B\rangle$. Clearly, since $T_{A} \equiv\left\langle A, A_{1}\right\rangle$, the equation of $T_{A}$ is simply $y=0$. Since $\mathbf{B}=\mathbf{A}+X \mathbf{A}_{1}+Y \mathbf{A}_{2}$, we have $B_{1}=X^{\prime} \mathbf{A}_{1}+Y^{\prime} \mathbf{A}_{2}$ where ' indicates the derivative with respect to arclength. Since $T_{B} \equiv\left\langle B, B_{1}\right)$, the equation of $T_{B}$ is $(X-x) Y^{\prime}+(y-Y) X^{\prime}=0$. Finally, the equation of $\langle A, B\rangle$ is $y X-x Y=0$. The equation of our pencil of conics can thus be written as:

$$
\left((X-x) Y^{\prime}+(y-Y) X^{\prime}\right) y+\lambda(y X-x Y)^{2}=0
$$


Let us now compute $\lambda_{A}$ and $\lambda_{B}$. To find $\lambda_{A}$, we rewrite (49) as:

$$
\begin{gathered}
y=-\frac{\lambda}{X Y^{\prime}-X^{\prime} Y-x Y^{\prime}+y X^{\prime}}(y X-x Y)^{2}= \\
-\frac{\lambda}{X Y^{\prime}-X^{\prime} Y}(y X-x Y)^{2}\left(1-\frac{-x Y^{\prime}+y X^{\prime}}{X Y^{\prime}-X^{\prime} Y}+\cdots\right.
\end{gathered}
$$

expanding the square we find:

$$
y=-\frac{\lambda Y^{2}}{X Y^{\prime}-X^{\prime} Y} x^{2}+\alpha x y+\beta y^{2}+\text { terms of higher degree in } x \text { and } y
$$

Since the reduced equation of the curve $(c)$ is $y=\frac{x^{2}}{2}+o\left(x^{4}\right)([4])$, the conic $\left(c_{A}\right)$ of the pencil which has a contact of order 3 with $(c)$ at $A$ is defined by:

$$
\lambda_{A}=\frac{X^{\prime} Y-X Y^{\prime}}{2 Y^{2}}
$$

The Frenet frame at $B$ is defined by $B$ and $B_{1}$ which we have already discussed and $B_{2}$ whose coordinates in the Frenet frame at $A$ are $X^{\prime \prime}$ and $Y^{\prime \prime}$. Thus the equation of the line $\left\langle B, B_{2}\right\rangle$ is $(X-x) Y^{\prime \prime}+(y-Y) X^{\prime \prime}=0$. We let $u=(X-x) Y^{\prime \prime}+(y-Y) X^{\prime \prime}$ and $v=(X-x) Y^{\prime}+(y-Y) X^{\prime}$. The equation of $(c)$ in the Frenet frame $\left(B, B_{1}, B_{2}\right)$ can be written $v=\frac{u^{2}}{2}+o\left(u^{4}\right)$. A straightforward computation shows that $x$ and $y$ can be expressed as functions of $u$ and $v$ as follows:

$$
x=X+\frac{v X^{\prime \prime}-u X^{\prime}}{Y^{\prime \prime} X^{\prime}-Y^{\prime} X^{\prime \prime}} \quad y=Y+\frac{v Y^{\prime \prime}-u Y^{\prime}}{Y^{\prime \prime} X^{\prime}-Y^{\prime} X^{\prime \prime}}
$$

This allows us to rewrite equation (49) as follows:

$$
v\left(Y+\frac{v Y^{\prime \prime}-u Y^{\prime}}{Y^{\prime \prime} X^{\prime}-Y^{\prime} X^{\prime \prime}}\right)+\lambda\left(\frac{v\left(X Y^{\prime \prime}-X^{\prime \prime} Y\right)+u\left(X^{\prime} Y-Y^{\prime} X\right)}{Y^{\prime \prime} X^{\prime}-Y^{\prime} X^{\prime \prime}}\right)^{2}
$$

from where it follows that:

$$
v=-\frac{\lambda}{Y\left(Y^{\prime \prime} X^{\prime}-Y^{\prime} X^{\prime \prime}\right)^{2}}\left(u^{2}\left(X^{\prime} Y-X Y^{\prime}\right)^{2}+\alpha u v+\beta v^{2}\right) \frac{1}{1+\frac{v Y^{\prime \prime}-u Y^{\prime}}{Y\left(Y^{\prime \prime} X^{\prime}-Y^{\prime} X^{\prime \prime}\right)}}
$$

and therefore:

$$
v=-\frac{\lambda\left(X^{\prime} Y-X Y^{\prime}\right)^{2}}{Y\left(Y^{\prime \prime} X^{\prime}-Y^{\prime} X^{\prime \prime}\right)^{2}} u^{2}+\gamma u v+\delta v^{2}+\text { terms of higher degree in } u \text { and } v
$$

Since the reduced equation of the curve $(c)$ is $v=\frac{u^{2}}{2}+o\left(u^{4}\right)$, the conic $\left(c_{B}\right)$ of the pencil which has a contact of order 3 with $(c)$ at $B$ is defined by:

$$
\lambda_{B}=-\frac{Y\left(Y^{\prime \prime} X^{\prime}-Y^{\prime} X^{\prime \prime}\right)^{2}}{2\left(X^{\prime} Y-X Y^{\prime}\right)^{2}}
$$

From this we write the ratio $\lambda_{A} / \lambda_{B}$ :

$$
\frac{\lambda_{A}}{\lambda_{B}}=\frac{\left(X Y^{\prime}-X^{\prime} Y\right)^{3}}{Y^{3}\left(Y^{\prime \prime} X^{\prime}-Y^{\prime} X^{\prime \prime}\right)^{2}}
$$

Considering the fact that we have $Y=\frac{X^{2}}{2}-\frac{X^{5}}{20}+o\left(X^{7}\right)$ (see for example [4]), we find that:

$$
\log ^{1 / 3}\left(\frac{\lambda_{A}}{\lambda_{B}}\right)=\left(\frac{11}{10}\right)^{1 / 3} X+o\left(X^{2}\right)
$$

Since $X=\sigma+O\left(\sigma^{2}\right)([4])$, we have obtained the following important result: 
Proposition 5.1 Let $A$ and $B$ be two points of a curve (c) and $\sigma$ be the projective arc-length of the portion of the curve between $A$ and $B$. If we consider the four conics $\left(c_{1}\right),\left(c_{2}\right),\left(c_{A}\right),\left(c_{B}\right)$ defined above and belonging to the pencil of conics tangent in $A$ to the tangent to (c) at $A$ and in $B$ to the tangent to $(c)$ at $B$, we can define their cross-ratio $\tau=\left\{\left(c_{1}\right),\left(c_{2}\right) ;\left(c_{A}\right),\left(c_{B}\right)\right\}$. We have the following property:

$$
\lim _{\sigma \rightarrow 0} \frac{\log ^{1 / 3} \tau}{\sigma}=\left(\frac{11}{10}\right)^{1 / 3}
$$

This proposition gives a geometric interpretation of the projective arclength.

\subsection{Analytic determination of the arc length parameter and projective curvature}

We shall use a method which is somewhat different from the affine case, i.e., the method of differential equations. This gives a complementary insight, even if the moving frame theory is more geometrical. We thus consider a point $A$ of a plane curve $(c)$ parametrized by $t$ with projective coordinates $x_{0}(t), x_{1}(t), x_{2}(t)$. We let $\mathbf{A}=\left[x_{0}(t), x_{1}(t), x_{2}(t)\right]^{T}$. The coordinates $x_{i}$ of $A$ all satisfy the same third order differential equation obtained by setting the determinant of order 4

$$
\left|\begin{array}{llll}
\theta^{\prime \prime \prime} & \theta^{\prime \prime} & \theta^{\prime} & \theta \\
\mathbf{A}^{\prime \prime \prime} & \mathbf{A}^{\prime \prime} & \mathbf{A}^{\prime} & \mathbf{A}
\end{array}\right|
$$

to 0 (take $\theta=x_{i}, i=0,1,2$ ).

We write this equation in the following form

$$
\theta^{\prime \prime \prime}+p \theta^{\prime \prime}+q \theta^{\prime}+r \theta=0
$$

by letting

$$
p=-\frac{\left|\begin{array}{lll}
\mathbf{A}^{\prime \prime \prime} & \mathbf{A}^{\prime} & \mathbf{A}
\end{array}\right|}{\left|\begin{array}{lll}
\mathbf{A}^{\prime \prime} & \mathbf{A}^{\prime} & \mathbf{A}
\end{array}\right|} q=\frac{\left|\begin{array}{lll}
\mathbf{A}^{\prime \prime \prime} & \mathbf{A}^{\prime \prime} & \mathbf{A}
\end{array}\right|}{\left|\begin{array}{lll}
\mathbf{A}^{\prime \prime} & \mathbf{A}^{\prime} & \mathbf{A}
\end{array}\right|} \quad r=-\frac{\left|\begin{array}{lll}
\mathbf{A}^{\prime \prime \prime} & \mathbf{A}^{\prime \prime} & \mathbf{A}^{\prime}
\end{array}\right|}{\left|\begin{array}{lll}
\mathbf{A}^{\prime \prime} & \mathbf{A}^{\prime} & \mathbf{A}
\end{array}\right|}
$$

and excluding the points of inflexion for which

$$
\left|\begin{array}{lll}
\mathbf{A}^{\prime \prime} & \mathbf{A}^{\prime} & \mathbf{A}
\end{array}\right|=0
$$

Equation (52) defines a class of curves projectively equal to each other and to the curve (c), but it does not necessarily give all curves projectively equal to $(c)$. In effect, if we change $\mathbf{A}$ into $\overline{\mathbf{A}}=\lambda(t) \mathbf{A}$, we obtain a new equation (52) which, from the same curve (c), also defines curves that are projectively equal to it. This also applies to the change of parameter $t \rightarrow \bar{t}=f(t)$.This indeterminacy allows us to simplify the equation (52). We look for two functions $\lambda(t)$ and $f(t)$ in order to eliminate the terms in $\theta^{\prime}$ and $\theta^{\prime \prime}$ in the equation. We shall see that there are infinitely many possible ways, as $\bar{t}$ is only defined up to homography. 


\subsubsection{Reducing the differential equation}

We thus want to convert the equation

$$
\frac{d^{3} \mathbf{A}}{d t^{3}}+p \frac{d^{2} \mathbf{A}}{d t^{2}}+q \frac{d \mathbf{A}}{d t}+r \mathbf{A}=\mathbf{0}
$$

to the form

$$
\frac{d^{3} \overline{\mathbf{A}}}{d \bar{t}^{3}}+\bar{r} \overline{\mathbf{A}}=\mathbf{0}
$$

by means of the transformations

$$
\begin{aligned}
\overline{\mathbf{A}} & =\lambda(t) \mathbf{A} \\
\bar{t} & =f(t)
\end{aligned}
$$

In the following, we represent derivatives in $t$ by superscripts. We easily compute the following expressions

$$
\begin{aligned}
\frac{d \overline{\mathbf{A}}}{d \bar{t}}=\frac{d \overline{\mathbf{A}}}{d t} \frac{d t}{d \bar{t}}= & \frac{\lambda^{\prime}}{f^{\prime}} \mathbf{A}+\frac{\lambda}{f^{\prime}} \mathbf{A}^{\prime}, \\
\frac{d^{2} \overline{\mathbf{A}}}{d \bar{t}^{2}}= & \left(\frac{\lambda^{\prime \prime}}{f^{\prime 2}}-\frac{\lambda^{\prime} f^{\prime \prime}}{f^{\prime 3}}\right) \mathbf{A}+\left(\frac{2 \lambda^{\prime}}{f^{\prime 2}}-\frac{\lambda f^{\prime \prime}}{f^{\prime 3}}\right) \mathbf{A}^{\prime}+\frac{\lambda}{f^{\prime 2}} \mathbf{A}^{\prime \prime}, \\
\frac{d^{3} \overline{\mathbf{A}}}{d \bar{t}^{3}}+\bar{r} \overline{\mathbf{A}}= & \left(\frac{\lambda^{\prime \prime \prime}}{f^{\prime 3}}-\frac{3 \lambda^{\prime \prime} f^{\prime \prime}}{f^{\prime 4}}-\frac{\lambda^{\prime} f^{\prime \prime \prime}}{f^{\prime 4}}+\frac{3 \lambda^{\prime} f^{\prime \prime 2}}{f^{\prime 5}}+\bar{r} \lambda\right) \mathbf{A} \\
& +\left(\frac{3 \lambda^{\prime \prime}}{f^{\prime 3}}-\frac{6 \lambda^{\prime} f^{\prime \prime}}{f^{\prime 4}}-\frac{\lambda f^{\prime \prime \prime}}{f^{\prime 4}}+\frac{3 \lambda f^{\prime \prime 2}}{f^{\prime 5}}\right) \mathbf{A}^{\prime} \\
& +\left(\frac{3 \lambda^{\prime}}{f^{\prime 3}}-\frac{3 \lambda f^{\prime \prime}}{f^{\prime 4}}\right) \mathbf{A}^{\prime \prime}+\frac{\lambda}{f^{\prime 3}} \mathbf{A}^{\prime \prime \prime} .
\end{aligned}
$$

Thus in order to determine $\bar{r}(t), \lambda(t), f(t)$ we obtain the system of three equations

$$
\begin{aligned}
\frac{3 \lambda^{\prime}}{\lambda}-\frac{3 f^{\prime \prime}}{f^{\prime}} & =p \\
\frac{3 \lambda^{\prime \prime}}{\lambda}-\frac{6 \lambda^{\prime}}{\lambda} \frac{f^{\prime \prime}}{f^{\prime}}-\frac{f^{\prime \prime \prime}}{f^{\prime}}+\frac{3 f^{\prime \prime 2}}{f^{\prime 2}} & =q \\
\frac{\lambda^{\prime \prime \prime}}{\lambda}-\frac{3 \lambda^{\prime \prime}}{\lambda} \frac{f^{\prime \prime}}{f^{\prime}}-\frac{\lambda^{\prime}}{\lambda} \frac{f^{\prime \prime \prime}}{f^{\prime}}+\frac{3 \lambda^{\prime}}{\lambda} \frac{f^{\prime \prime 2}}{f^{\prime 2}}+\bar{r} f^{\prime 3} & =r
\end{aligned}
$$

The first equation gives the ratio $\frac{\lambda^{\prime}}{\lambda}$

$$
\frac{\lambda^{\prime}}{\lambda}-\frac{f^{\prime \prime}}{f^{\prime}}=\frac{p}{3}
$$

hence the ratio $\frac{\lambda^{\prime \prime}}{\lambda}$ by derivation. If we replace these two ratios by their expressions in the second equation after simplifying, we obtain

$$
\frac{1}{2} \frac{f^{\prime \prime \prime}}{f^{\prime}}-\frac{3}{4} \frac{f^{\prime \prime 2}}{f^{\prime 2}}=-\frac{1}{12} p^{2}-\frac{1}{4} p^{\prime}+\frac{1}{4} q
$$


The first member of this equation is the Schwarzian of $\bar{t}$ with respect to $t$. Once $\bar{t}$ is known we deduce $\lambda$ by means of (55).

We easily see that $\bar{t}$ is defined only up to homography. In effect we have the following proposition

Proposition 5.2 If we consider two functions $f(t)$ and $F(t)$ of the same variable $t$, then a necessary and sufficient condition for $F$ and $f$ to be related by a homographic relation with fixed coefficients

$$
F(t)=\frac{a f(t)+b}{c f(t)+d}
$$

is that the Schwarzians $\{F\}_{t}$ and $\{f\}_{t}$ are equal.

Proof : The proof involves eliminating the three unknown parameters defining the homographic transformation by successive derivations. We first obtain

$$
F^{\prime}(t)=\frac{(a d-b c) f^{\prime}(t)}{(c f(t)+d)^{2}}
$$

and the logarithmic derivative of both sides of this equation gives

$$
\frac{F^{\prime \prime}(t)}{F^{\prime}(t)}=\frac{f^{\prime \prime}(t)}{f^{\prime}(t)}-\frac{2 f^{\prime}(t)}{f(t)+\frac{d}{c}}
$$

A further derivation eliminates the constant $\frac{d}{c}$ giving the equation

$$
\frac{F^{\prime \prime \prime}(t)}{F^{\prime}(t)}-\frac{3}{2} \frac{F^{\prime \prime 2}(t)}{F^{\prime 2}(t)}=\frac{f^{\prime \prime \prime}(t)}{f^{\prime}(t)}-\frac{3}{2} \frac{f^{\prime \prime 2}(t)}{f^{\prime 2}(t)}
$$

i.e., as required

$$
\{F\}_{t}=\{f\}_{t}
$$

It follows that if we consider the cross-ratio of the four values of $\bar{t}$ corresponding to any four points of $(c)$, this ratio does not depend on the particular choice of $\bar{t}$. This is an invariant for the four point system. We shall call it the four point cross-ratio on $(c)$. It is the analog of the ratio of the affine arc length parameters of three points in the case of the affine group in section 4.7 .

\subsubsection{Projective arc length parameter}

If we take into account the expressions of $\bar{t}$ and of $\lambda(t)$ in the last equation of the previous system, this reduces to

$$
\bar{r} f^{\prime 3}=r-\frac{1}{3} p q+\frac{2}{27} p^{3}-\frac{1}{2} q^{\prime}+\frac{1}{3} p p^{\prime}+\frac{1}{6} p^{\prime \prime} \equiv H(t)
$$

which can also be written

$$
\bar{r} d \bar{t}^{3}=H d t^{3}
$$

Thus whatever the choice of $\bar{t}$ as the solution of the Schwarz equation, the expression of $\bar{r} d \bar{t}^{3}$ always has the same value. We obtain the differential $d \sigma$ of the arc length parameter by letting

$$
d \sigma^{3}=\bar{r} d \bar{t}^{3}=H d t^{3}
$$


Thus we have

$$
d \sigma=\bar{r}^{\frac{1}{3}} d \bar{t}=H^{\frac{1}{3}} d t
$$

Since $H$ involves derivatives of the fifth order, we find that the differential of the projective arc length parameter is an invariant of $(c)$ of the fifth order.

\subsubsection{Projective curvature}

Using $\sigma$ to parametrize $(c)$, equation (53) has no terms in $\frac{d^{2} \mathrm{~A}}{d \sigma^{2}}$ and is written in the form

$$
\frac{d^{3} \mathbf{A}}{d \sigma^{3}}+2 k \frac{d \mathbf{A}}{d \sigma}+h \mathbf{A}=\mathbf{0}
$$

The quantities $h$ and $k$ are invariant by a projective transformation on (c), but they are not independent since, for any parameter $t$, we must have

$$
d \sigma^{3}=H d t^{3}
$$

In particular, if we have $d t=d \sigma$, we have $H=1$ which, taking into account equation (57) enables us to write

$$
h-k^{\prime}=1
$$

When parametrized by its projective arc length, the differential equation of the curve is written in the form

$$
\frac{d^{3} \mathbf{A}}{d \sigma^{3}}+2 k \frac{d \mathbf{A}}{d \sigma}+\left(k^{\prime}+1\right) \mathbf{A}=\mathbf{0}
$$

This leaves only one invariant $k$ in the equation, and it is the projective curvature introduced previously. As $\sigma$ is the chosen parameter, we can compute the projective curvature, and

$$
t=\sigma \quad p=0 \quad q=2 k \quad r=k^{\prime}+1
$$

The Schwarzian $\{\bar{t}\}_{\sigma}$ of $\bar{t}$ with respect to $\sigma$ is given by (equation (56))

$$
\{\bar{t}\}_{\sigma}=\frac{1}{2} k
$$

and we can deduce

$$
k=2\{\bar{t}\}_{\sigma}
$$

To determine $k$ we need the following theorem

Theorem 5.1 Given two functions $x(t)$ and $y(t)$ of the same variable $t$, we have

$$
\{y\}_{x} d x^{2}=\left[\{y\}_{t}-\{x\}_{t}\right] d t^{2}
$$


Applying this theorem to $x(t)=\sigma(t)$ and $y(t)=\bar{t}(t)$, we obtain

$$
\{\bar{t}\}_{\sigma} d \sigma^{2}=\left[\{\bar{t}\}_{t}-\{\sigma\}_{t}\right] d t^{2}
$$

hence, taking into account the expression of $k$, we have

$$
k=2 \frac{\{\bar{t}\}_{t}-\{\sigma\}_{t}}{\frac{d \sigma^{2}}{d t}}
$$

We know $\{\bar{t}\}_{t}$ and we need to compute $\{\sigma\}_{t}$. We have

$$
\frac{d \sigma}{d t}=H^{\frac{1}{3}}
$$

Deriving logarithmically

$$
\frac{\sigma^{\prime \prime}}{\sigma^{\prime}}=\frac{1}{3} \frac{H^{\prime}}{H}
$$

and a further derivative yields

$$
\frac{\sigma^{\prime \prime \prime}}{\sigma^{\prime}}-\frac{\sigma^{\prime \prime 2}}{\sigma^{\prime 2}}=\frac{1}{3} \frac{H^{\prime \prime}}{H}-\frac{1}{3} \frac{H^{\prime 2}}{H^{2}}
$$

From these two relations we immediately deduce

$$
\{\sigma\}_{t}=\frac{1}{2} \frac{\sigma^{\prime \prime \prime}}{\sigma^{\prime}}-\frac{3}{4} \frac{\sigma^{\prime \prime 2}}{\sigma^{\prime 2}}=\frac{1}{6} \frac{H^{\prime \prime}}{H}-\frac{7}{36} \frac{H^{\prime 2}}{H^{2}}
$$

Hence by replacement in the expression of $k$

$$
k=H^{-\frac{2}{3}}\left[-\frac{1}{2} p^{\prime}-\frac{1}{6} p^{2}+\frac{1}{2} q-\frac{1}{3} \frac{H^{\prime \prime}}{H}+\frac{7}{18} \frac{H^{\prime 2}}{H^{2}}\right]
$$

\subsubsection{Relation with unimodal affine geometry}

Let us suppose that the curve (c) is

parametrized by its affine arc length $\sigma_{a}$. The affine Frenet frame is a special case of the projective frame where the vectors $e_{1}$ and $e_{2}$ represent $A_{1}$ and $A_{2}$, two points of the line at infinity, whose projective coordinates are $\mathbf{A}_{i}=\left[\mathbf{e}_{i}^{T}, 0\right]^{T}, i=1,2$. Also, the origin $A_{a}$ of the frame defines a projective point $A_{p}$ with coordinates $\mathbf{A}_{p}=\left[\mathbf{A}_{a}^{T}, 1\right]^{T}$. According to the affine Frenet equations (33), the point $A\left(\sigma_{a}\right)$ satisfies the differential equation

$$
\left|\begin{array}{cccc}
\theta^{\prime \prime \prime} & \theta^{\prime \prime} & \theta^{\prime} & \theta \\
k_{a} \mathbf{e}_{1} & \mathbf{e}_{2} & \mathbf{e}_{1} & \mathbf{A}_{a} \\
0 & 0 & 0 & 1
\end{array}\right|=0
$$

written in the form

$$
\theta^{\prime \prime \prime}-k_{a} \theta^{\prime}=0
$$

where $k_{a}$ is the affine curvature. We thus have

$$
p=0 \quad q=-k_{a} \quad r=0
$$


The differential equation that gives the projective parameter $\bar{t}$ (equation (56)) is

$$
\{\bar{t}\}_{\sigma_{a}}=-\frac{1}{4} k_{a}
$$

As for the projective arc length $\sigma_{p}$ (equation (57)), we have

$$
H\left(\sigma_{a}\right)=\frac{1}{2} \frac{d k_{a}}{d \sigma_{a}}
$$

By making this equation closer to the equation (60) we obtain the relation between the projective curvature $k_{p}$ and the affine curvature

$$
k_{p}=\left(\frac{k_{a}^{\prime}}{2}\right)^{-\frac{2}{3}}\left[-\frac{k_{a}}{2}-\frac{1}{3} \frac{k_{a}^{\prime \prime \prime}}{k_{a}^{\prime}}+\frac{7}{18}\left(\frac{k_{a}^{\prime \prime}}{k_{a}^{\prime}}\right)^{2}\right]
$$

where' indicate a derivative in $\sigma_{a}$. We also obtain the relation between the projective arc length and the affine arc length

$$
\frac{d \sigma_{p}}{d \sigma_{a}}=\left(\frac{1}{2} \frac{d k_{a}}{d \sigma_{a}}\right)^{\frac{1}{3}}
$$

We can also consider the scale factor $\lambda$, a function of $\sigma_{p}$, which enables us to transform from the affine point $A_{a}$ to the normalized point of the projective Frenet frame. We write that $\mathbf{A}=\lambda \mathbf{A}_{a}$ and we determine $\lambda$ so that $\left|\begin{array}{lll}\mathbf{A} & \mathbf{A}_{1} & \mathbf{A}_{2}\end{array}\right|=1$. We then apply the affine and projective Frenet formulas

$$
\begin{array}{lcc}
\mathbf{A}_{1} & =\lambda^{\prime} \mathbf{A}_{a} & +\lambda \frac{d \sigma_{a}}{d \sigma_{p}} \mathbf{e}_{1} \\
\mathbf{A}_{2}=\left(\lambda^{\prime \prime}+\lambda k\right) \mathbf{A}_{a} & +\left(2 \lambda^{\prime} \frac{d \sigma_{a}}{d \sigma_{p}}+\lambda \frac{d^{2} \sigma_{a}}{d \sigma_{p}^{2}}\right) \mathbf{e}_{1}+\lambda\left(\frac{d \sigma_{a}}{d \sigma_{p}}\right)^{2} k_{a} \mathbf{e}_{2}
\end{array}
$$

where ' indicates a derivative in $\sigma_{p}$ and we find

$$
\left|\begin{array}{lll}
\mathbf{A} & \mathbf{A}_{1} & \mathbf{A}_{2}
\end{array}\right|=\lambda^{3}\left(\frac{d \sigma_{a}}{d \sigma_{p}}\right)^{3}=1
$$

Hence, given the equation (62),

$$
\lambda=\left(\frac{k_{a}^{\prime}}{2}\right)^{\frac{1}{3}}=\frac{d \sigma_{p}}{d \sigma_{a}}
$$

where the ' indicates a derivative in $\sigma_{a}$.

\subsection{Application to the evolution of curves}

Similarly to the euclidean and affine cases (see sections 3.3 and 4.8), we are now going to consider the case of a family of curves. We use the same notations as in these sections and consider smooth embedded plane curves deforming in time. Let $\mathbf{A}(p, t): \mathcal{S}^{1} \times R \rightarrow \mathcal{P}^{2}$ be a family of such curves where $p$ parametrizes the curve and $t$ represents the time. Let $\sigma$ be the projective arc-length along a curve of the family, a function of $p$ and $t$. We now propose to study the following evolution equation:

$$
\mathbf{A}_{\mathbf{t}}=\mathbf{A}_{\sigma \sigma}
$$


in which the partial with respect to $t$ is taken at $p$ constant and the partials with respect to $\sigma$ are taken at $t$ constant. This equation, as (24) and (41), can be thought of as a heat equation (because of the formal similarity with the usual heat equation) which is intrinsic to the curve. It has been studied by this author $[5,6]$.

Since a curve in the projective plane is defined up to an projective transformation by its arclength and curvature, it is natural to establish how they evolve in time when the curve changes according to (65). The key is of course to use the Frenet equations (48).

We use the equations (25), (26), (27) which are unchanged in the projective framework. We will also need the following expressions of the higher order derivatives of $\mathbf{A}$ with respect to the arc-length $\sigma$ which we obtain from the Frenet formulae:

$$
\begin{aligned}
& \mathbf{A}_{\sigma^{3}}=-2 k \mathbf{A}_{\sigma}-\left(1+k_{\sigma}\right) \mathbf{A} \\
& \mathbf{A}_{\sigma^{4}}=\left(-k_{\sigma^{2}}+2 k^{2}\right) \mathbf{A}-\left(1+3 k_{\sigma}\right) \mathbf{A}^{(1)}-2 k \mathbf{A}^{(2)} \\
& \mathbf{A}_{\sigma^{3}}=\left(-k_{\sigma^{3}}+7 k k_{\sigma}+3 k\right) \mathbf{A}+4\left(k^{2}-k_{\sigma^{2}}\right) \mathbf{A}^{(1)}-\left(1+5 k_{\sigma}\right) \mathbf{A}^{(2)}
\end{aligned}
$$

In these equations, we have written $\mathbf{A}^{(1)}$ and $\mathbf{A}^{(2)}$ instead of the usual $\mathbf{A}_{1}$ and $\mathbf{A}_{2}$ to avoid problems with partial derivatives.

\subsubsection{Evolution of projective arc-length}

It is now easy to characterize the evolution of arc-length. We use the relation

$$
\left|\begin{array}{ll}
\mathbf{A} & \mathbf{A}^{(1)} \\
\mathbf{A}^{(2)}
\end{array}\right|=1
$$

and take its derivative with respect to $t$ :

$$
\left|\mathbf{A} \quad \mathbf{A}^{(1)} \quad \mathbf{A}^{(2)}\right|_{t}=\left|\mathbf{A}_{t} \mathbf{A}^{(1)} \mathbf{A}^{(2)}\right|+\left|\mathbf{A} \mathbf{A}_{t}^{(1)} \mathbf{A}^{(2)}\right|+\left|\mathbf{A} \mathbf{A}^{(1)} \mathbf{A}_{t}^{(2)}\right|=0
$$

According to the equations (65) and (48) the first determinant is equal to $-k$. According to the equations (25), (66) and (48) we have

$$
\mathbf{A}_{t}^{(1)}=\mathbf{A}_{t \sigma}=-\frac{g_{t}}{g} \mathbf{A}^{(1)}+\mathbf{A}_{\sigma^{3}}=-\left(\frac{g_{t}}{g}+2 k\right) \mathbf{A}^{(1)}-\left(1+k_{\sigma}\right) \mathbf{A}
$$

and thus the second determinant of the right-hand side is equal to $-\left(\frac{g_{t}}{g}+2 k\right)$. Similarly, the second Frenet equation allows us to write

$$
\mathbf{A}_{t}^{(2)}=\frac{\partial}{\partial t}\left(k \mathbf{A}+\mathbf{A}_{\sigma \sigma}\right)=k_{t} \mathbf{A}+k \mathbf{A}_{\sigma \sigma}+\mathbf{A}_{t \sigma \sigma}
$$

Using equations (26) and (67), we can compute the coefficient of the term $\mathbf{A}^{(2)}$ in that expression which yields $-\left(2 \frac{g_{t}}{g}+k\right)$ for the value of the third determinant in the right-hand side of equation (69). Adding these three values and equating them to zero, we obtain

$$
\mathbf{A}_{t \sigma} \wedge \mathbf{A}_{\sigma \sigma}=\left(k-\frac{g_{t}}{g}\right) \mathbf{A}_{\sigma} \wedge \mathbf{A}_{\sigma \sigma}=k-\frac{g_{t}}{g}
$$

Similarly, using equations (26) and (43), we obtain

$$
g_{t}=-\frac{4}{3} k g
$$

which is the projective analog of (31) and (45). 


\subsubsection{Evolution of projective curvature}

The principle of the method is once again to use a differential equation that is satisfied by each curve of the family. We can write equation (66) as

$$
\mathbf{A}_{\sigma^{3}}+2 k \mathbf{A}_{\sigma}=-\left(1+k_{\sigma}\right) \mathbf{A}
$$

The two sides of this equation represent the same projective point., i.e. $A$. The projective point represented by the left-hand side follows the same curve, for a constant value of $p$, as the point $A$. This implies that the tangents must be the same, and therefore that the two vectors $\mathbf{A} \wedge \mathbf{A}_{\boldsymbol{t}}$ and $\mathbf{A} \wedge \frac{\partial}{\partial t}\left(\mathbf{A}_{\sigma^{3}}+2 k \mathbf{A}_{\sigma}\right)$ are parallel. Using equations (65) and the Frenet equations, it is easy to show that

$$
\mathbf{A} \wedge \mathbf{A}_{t}=\mathbf{A} \wedge \mathbf{A}^{(2)}
$$

In order to compute $\frac{\partial}{\partial t}\left(\mathbf{A}_{\sigma} s+2 k \mathbf{A}_{\sigma}\right)$ we use equations (25), (27) et (68) to obtain

$$
\mathbf{A} \wedge \frac{\partial}{\partial t}\left(\mathbf{A}_{\sigma^{3}}+2 k \mathbf{A}_{\sigma}\right)=\left(2 k_{t}-4 k_{\sigma^{2}}+4 k \frac{g_{t}}{g}-\left[\frac{g_{t}}{g}\right]_{\sigma^{2}}\right) \mathbf{A} \wedge \mathbf{A}^{(1)}-\left(1+5 k_{\sigma}+3\left[\frac{g_{t}}{g}\right]_{\sigma}\right) \mathbf{A} \wedge \mathbf{A}^{(2)}
$$

The condition that the two vectors are parallel is thus equivalent to the two equations

$$
\begin{gathered}
2 k_{t}-4 k_{\sigma^{2}}+4 k \frac{g_{t}}{g}-\left[\frac{a_{t}}{g}\right]_{\sigma^{2}}=0 \\
1+5 k_{\sigma}+3\left[\frac{g_{t}}{g}\right]_{\sigma}=\alpha
\end{gathered}
$$

where $\alpha$ is a function that is equal to $1+k_{\sigma}$ according to the equation (70). Replacing in the first equation the value of $\frac{g_{t}}{g}$ and of its second order derivative with respect to $\sigma$ computed from the second equation, we obtain the sought-for evolution equation:

$$
k_{t}=\frac{4}{3}\left(k_{\sigma^{2}}+2 k^{2}\right)
$$

which is the projective analog of (32) and (46).

\subsubsection{Evolution of the affine arc-length and curvature}

We now relate the previous results to affine geometry for two reasons. The first reason is that the analog of equation (65) has been studied in the affine case and therefore it is interesting to compare the evolutions of the curve in the two cases. the second reason is that, in some sense, the affine results will shed some light on the projective ones.

Let then $E_{i}, i=1, \cdots, 4$ be the standard projective basis of $\mathcal{P}^{2}$ which is represented by the standard basis of $R^{3}$ and the vector $[1,1,1]^{T}$. The set $\left.\mathcal{P}^{2}\right\rangle\left\langle E_{1}, E_{2}\right\rangle$ i.e. the projective plane minus the "line at infinity" is isomorphic to the affine plane $\mathcal{A}^{2}$. This identification allows to define a family of curves embedded in $\mathcal{A}^{2}$ from a family of curves embedded in $\mathcal{P}^{2}$.

Let $X, Y$ and $Z$ be the coordinates of the vector $\mathbf{A}$ representing the projective point $A$ belonging to one the curves in the family. Since we are interested in the part of the curve included in $\mathcal{A}^{2}$, we can assume that $Z \neq 0$. Let $\mathbf{a}=\frac{1}{Z} \mathrm{~A}$ be the representative of the point $A$ in $\mathcal{A}^{2}$. At each time instant $t$, we can assume that this point is parametrized by its projective arc-length $\sigma_{p}$. From section 4 we know that we can define an affine arc-length as

$$
\frac{d \sigma_{a}}{d \sigma_{p}}=\left|\mathbf{E}_{3}, \mathbf{a}_{\sigma_{p}}, \mathbf{a}_{\sigma_{p} \sigma_{p}}\right|^{\frac{1}{3}}
$$


if we stay away from the inflection points where $a_{\sigma_{p}} \wedge a_{\sigma_{p} \sigma_{p}}=0$. We use the affine Frenet equations (33) in

$$
\begin{aligned}
& \frac{d \mathbf{a}}{d \sigma_{a}}=\mathbf{e}_{1} \\
& \frac{d \mathbf{e}_{1}}{d \sigma_{a}}=\mathbf{e}_{2} \\
& \frac{d \mathbf{e}_{2}}{d \sigma_{a}}=k_{a} \mathbf{e}_{1}
\end{aligned}
$$

We consider the vectors $e_{1}$ and $e_{2}$ as vectors of $R^{3}$ whose last coordinate is 0 to be compatible with our study of $\mathcal{P}^{2}$. The vectors $\mathbf{e}_{1}$ and $\mathbf{e}_{2}$ are related by

$$
\mathbf{e}_{1} \wedge \mathbf{e}_{2}=\mathbf{E}_{3}
$$

We have also derived in a previous section the following relations between the affine and projective Frenet frames (equations (63))

$$
\begin{aligned}
& \mathbf{A}^{(1)}=Z_{\sigma_{p}} \mathbf{a}+Z \frac{d \sigma_{a}}{d \sigma_{p}} \mathbf{e}_{1} \\
& \mathbf{A}^{(2)}=\left(Z_{\sigma_{p} \sigma_{p}}+Z k_{p}\right) \mathbf{a}+\left(2 Z_{\sigma_{p}} \frac{d \sigma_{a}}{d \sigma_{p}}+Z \frac{d^{2} \sigma_{a}}{d \sigma_{p}^{2}}\right) \mathbf{e}_{1}+Z\left(\frac{d \sigma_{a}}{d \sigma_{p}}\right)^{2} \mathbf{e}_{2}
\end{aligned}
$$

and (equation (64))

$$
Z=\frac{d \sigma_{p}}{d \sigma_{a}}
$$

If we now consider for convenience the quantity

$$
\Lambda=\frac{1}{2} \frac{d k_{a}}{d \sigma_{a}}
$$

we can prove (see $[5,6]$ for details) the following two results

Proposition 5.3 Let $h=\frac{d \sigma_{a}}{d p}$, the temporal evolution of the affine arc-length is

$$
h_{t}=-\frac{h}{3}\left(4 k_{p}+\Lambda^{-\frac{2}{3}}\left(\frac{\Lambda_{\sigma_{a} \sigma_{a}}}{\Lambda}-\left(\frac{\Lambda_{\sigma_{a}}}{\Lambda}\right)^{2}\right)\right.
$$

and

Proposition 5.4 The function $\Lambda$ evolves according to the equation

$$
\Lambda_{t}=\Lambda^{\frac{1}{3}}\left[\frac{\Lambda_{\sigma_{a} \sigma_{a}}}{\Lambda}-\left(\frac{\Lambda_{\sigma_{a}}}{\Lambda}\right)^{2}\right]=\Lambda^{\frac{1}{3}}\left[\log \mid \Lambda \|_{\sigma_{a} \sigma_{a}}\right.
$$

Moreover, if we perform the change of variable $V=\frac{1}{3} \log \Lambda^{2}, V$ evolves according to the equation

$$
V_{t}=e^{-V} V_{\sigma_{a} \sigma_{a}}
$$


Looking back at equation (61) giving the relation between the projective and affine curvatures, we see that equation (77) allows to compute the ratio $\frac{k_{a}^{\prime \prime \prime}}{k_{a}^{\prime}}=\frac{\Lambda_{\sigma_{a} \sigma_{a}}}{\Lambda}$ which involves a third order derivative of the affine curvature with respect to the affine arc length as a function of the second order derivatives of the affine curvature with respect to the affine arc length and the time parameter of the projective evolution equation (65). Thus, just as in the affine case we gain one order of derivation if we trade space (arc length) for time (scale).

\section{Conclusion}

This article is a general introduction to Cartan's moving frame method which is elegant, simple, and of an algorithmic nature. We have demonstrated how to use it systematically on three examples relevant to computer vision, curve in the euclidean, affine and projective planes, and derived the corresponding Frenet equations. We have then used these equations to show that the analysis of the deformation of plane curves according to an intrinsic heat equation could be done in a common framework, yielding very similar expressions for the evolution of the three curvature invariants.

\section{References}

[1] Luis Alvarez, Frédéric Guichard, Pierre-Louis Lions, and Jean-Michel Morel. Axioms and Fundamental Equations of Image Processing. Technical Report 9231, CEREMADE, 1992.

[2] Elie Cartan. La Théorie des Groupes Finis et Continus et la Géométrie Différentielle traitée par la Méthode du Repère Mobile. Jacques Gabay, 1992. Original edition, Gauthiers-Villars, 1937.

[3] Elie Cartan. Leçons sur la Théorie des Espaces à Connexion Projective. Jacques Gabay, 1992. Original edition, Gauthiers-Villars, 1937.

[4] O.D. Faugeras. Géométrie affine et projective en vision par ordinateur : I le cas des courbes. Technical report, INRIA, 1993. To appear.

[5] Olivier Faugeras. On the evolution of simple curves of the real projective plane. Comptes rendus de l'Académie des Sciences de Paris, Tome 317, Série I, (6):565-570, September 1993. Also INRIA Technical report number 1998.

[6] Olivier Faugeras. On the evolution of simple curves of the real projective plane. Technical Report 1998, INRIA, 1993.

[7] L. M. J. Florack, B. M. ter Haar Romeny, J. J. Koenderink, and M. A. Viergever. Scale and the differential structure of images. Image and Vision Computing, 10:376-388, July/August 1992.

[8] M. Gage. An isometric inequality with applications to curve shortening. Duke Mathematical Journal, 50:1225-1229, 1983.

[9] M. Gage. Curve shortening makes convex curves circular. Invent. Math., 76:357-364, 1984. 
[10] M. Gage and R.S. Hamilton. The heat equation shrinking convex plane curves. J. of Differential Geometry, 23:69-96, 1986.

[11] M. Grayson. The heat equation shrinks embedded plane curves to round points. J. of Differential Geometry, 26:285-314, 1987.

[12] Benjamin B. Kimia, Allen Tannenbaum, and Steven W. Zucker. On the Evolution of Curves via a Function of Curvature. I. The Classical Case. Journal of Mathematical Analysis and Applications, 163(2):438-458, 1992.

[13] Jan J. Koenderink. Solid Shape. MIT Press, 1990.

[14] J.J. Koenderink and A.J. van Doorn. Dynamic shape. Biological Cybernetics, 53:383-396, 1986.

[15] A. Mackworth and F. Mokhtarian. Scale-Based description and recognition of planar curves and two-dimensional shapes. IEEE Transactions on Pattern Analysis and Machine Intelligence, 8(1), January 1986.

[16] Joseph L. Mundy and Andrew Zimmerman, editors. Geometric Invariance in Computer Vision. MIT Press, 1992.

[17] A.H. Salden, B.M. ter Haar Romeny, and M. Viergever. Affine and projective differential geometric invariants of space curves. In Baba Vemuri, editor, Geometric Methods in Computer Vision II, pages 60-74. SPIE, July 1993.

[18] Guillermo Sapiro and Allen Tannenbaum. Affine shortening of Non-Convex Plane Curve. Technical Report EE PUB 845, Technion Israel Institute of Technology-Haifa, August 1992.

[19] Guillermo Sapiro and Allen Tannenbaum. On Affine Plane Curve Evolution. Technical Report EE PUB 821, Technion Israel Institute of Technology-Haifa, February 1992.

[20] Guillermo Sapiro and Allen Tannenbaum. Affine Invariant Scale Space. The International Journal of Computer Vision, 11(1):25-44, August 1993.

[21] Guillermo Sapiro and Allen Tannenbaum. On Invariant Curve Evolution and Image Analysis. Indiana University Journal of Mathematics, 1993. To appear.

[22] Joel Smoller. Shock Waves and Reaction-diffusion Equations. Springer-Verlag, New-York, 1983.

[23] A.P. Witkin. Scale-space filtering. In Proceedings of the International Joint Conference on Artificial Conference, pages 1019-1021, 1983. 


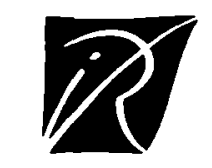

Unité de Recherche INRIA Sophia Antipolis

2004, route des Lucioles - B.P. 93 - 06902 SOPHIA ANTIPOLIS Cedex (France)

Linite de Recherche INRIA Lorraine Technopole de Nancy-Brabois - Campus Scientifique 615. rue du Jardin Botanique - B.P. 101 - 54602 VILLERS L.ES NANCY Cedex (France)

linire de Recherche INRIA Rentes IRISA. Campus Liniversitaire de Betalieu 35042 RFN NF.S Cedex (France)

bnite de Recherche INRIA Rhone-Alpes 46, avenue Félix Viallet - 3803l GRENOBL.E Cedex (France)

Linie de Recherche INRIA Rocquencourt Domaine de Volucedu Rocquencourt - B.P. $105-78153$ LE CHES.VAY Cedex (France)

EDITELR

INRIA - Domaine de Voluceau - Rocquencourt - B.P. 105 - 78153 LE CHESNAY Cedex (France)

ISSN $0249-6399$ 